\title{
LA-UR-17-20383
}

Approved for public release; distribution is unlimited.

Title: $\quad$ FleCSI: Comprehensive Slide Deck

Author(s): $\quad$ Bergen, Benjamin Karl

Intended for: General purpose slide deck of useful slides for various applications, i.e., conference presentation, online documentation, and reports.

Issued: 
Disclaimer:

Los Alamos National Laboratory, an affirmative action/equal opportunity employer, is operated by the Los Alamos National Security, LLC for the National Nuclear Security Administration of the U.S. Department of Energy under contract DE-AC52-06NA25396. By approving this article, the publisher recognizes that the U.S. Government retains nonexclusive, royalty-free license to publish or reproduce the published form of this contribution, or to allow others to do so, for U.S. Government purposes. Los Alamos National Laboratory requests that the publisher identify this article as work performed under the auspices of the U.S. Department of Energy. Los Alamos National Laboratory strongly supports academic freedom and a researcher's right to publish; as an institution, however, the Laboratory does not endorse the viewpoint of a publication or guarantee its technical correctness. 


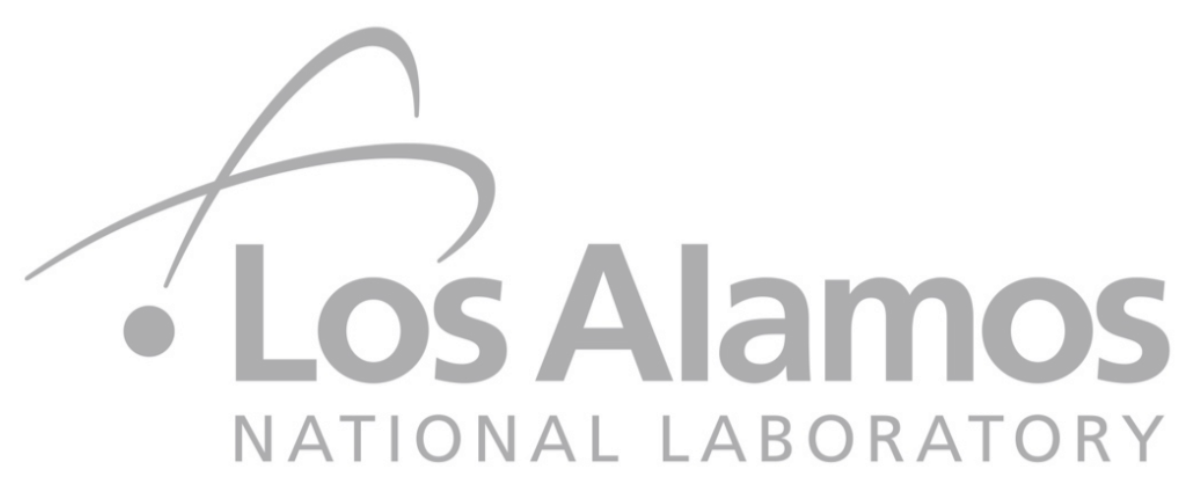




\section{What is FleCSI}




\section{What problems are we trying to solve?}

- Insulate production code development from uncertainty of evolving runtimes and architectures

- Focus: Data Model and Execution Model

- Allow basic interoperability of multiple physics packages

- Composability

- Allow project decomposition (no heroes...)

- Separation of concerns: modularity of design, complementary work

- Specialization within disciplines: professional roles 


\section{What problems are we trying to solve?}

- Provide basic mesh infrastructure (initial focus)

- Allow iterability of distributed-parallel collections

- Allow expression of topological relationships

- Ability to reason about these relationships statically (compile-time)

- Provide other infrastructure

- Graph and tree-based data structures

- Coloring and partitioning capabilities

- Dependency closures 


\section{What challenges are we trying to address with our CS development strategy?}

\section{Portability}

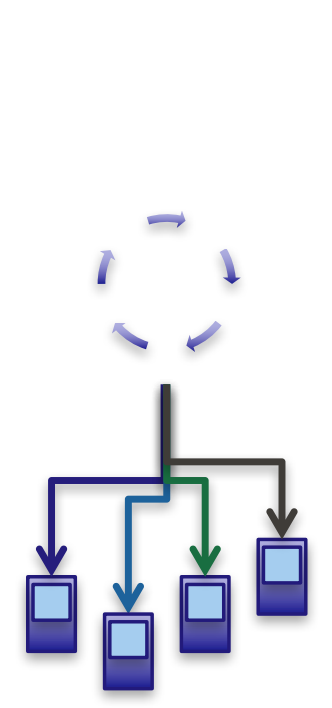

Dynamic Load Balancing

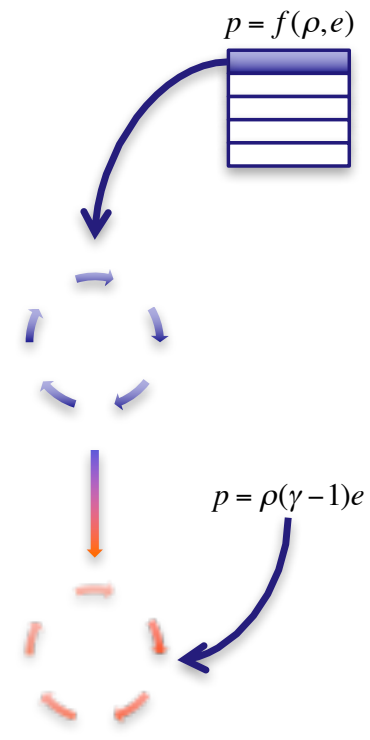

State-Dependent Models

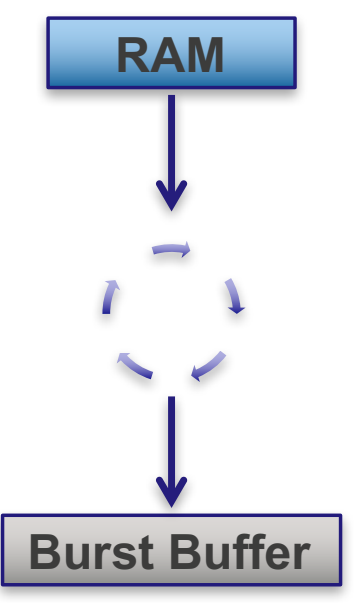

In-situ/In-transit Analysis

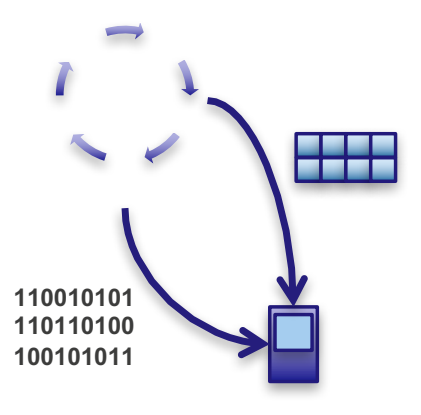

Kernel Specialization 


\section{What is FleCSI?}

FleCSI is a C++ programming system for developing multi-physics simulation codes...

- Runtime abstraction layer...

- High-level user interface, mid-level static specialization, low-level building blocks, tasking and fine-grained threading back-ends

- Programming model...

- Control, execution, and data models

- Useful data structure support...

- Mesh, N-Tree (N=3 $\rightarrow$ Octree), and KD-Tree

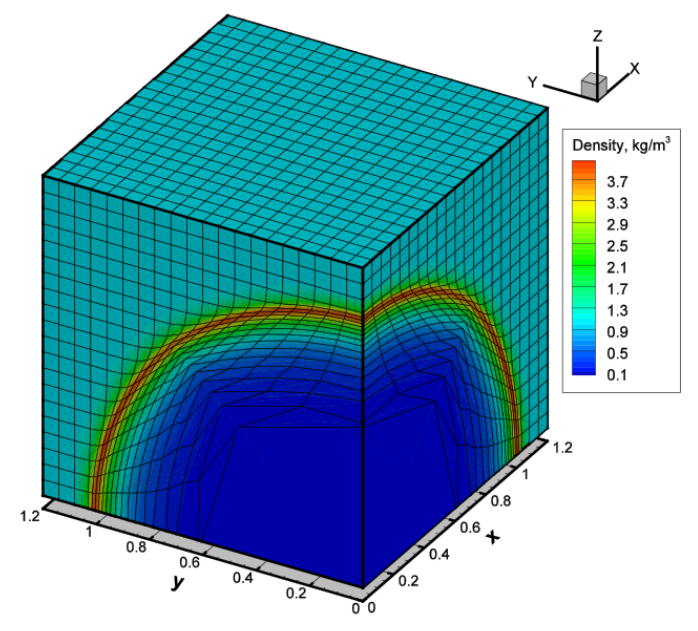

FleCSI: Pure 3D Lagrangian Sedov

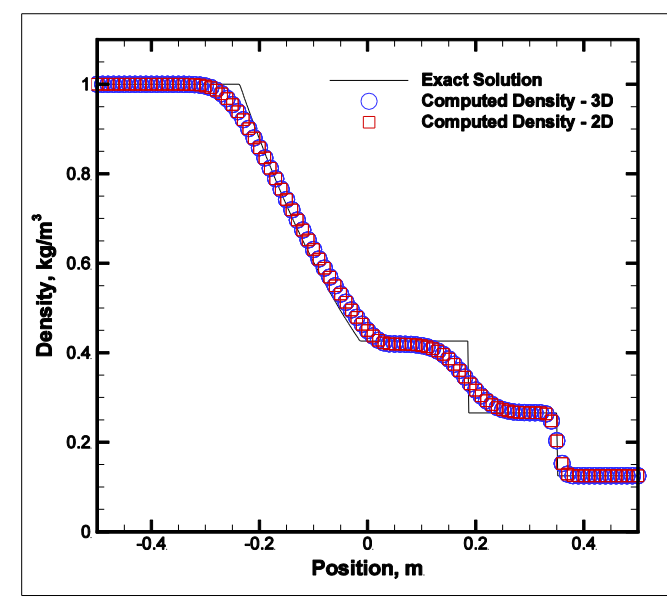

FleCSI: 2D/3D Eulerian Sod 


\section{Tree Data Structures}

\section{- Tree Topology}

- Hashed $n$-tree support (dimension independent: octtree, quadtree, etc.)

- Constant-time neighbor look-up

- Refinement and coarsening

- Morton ordering

- Tree traversal predicated with filter function

- Applications: SPH, N-Body, AMR, Complex Flows, Monte Carlo, Molecular Dynamics

- Collaborations with Mesh-Free and Transport teams will help us to mature this capability
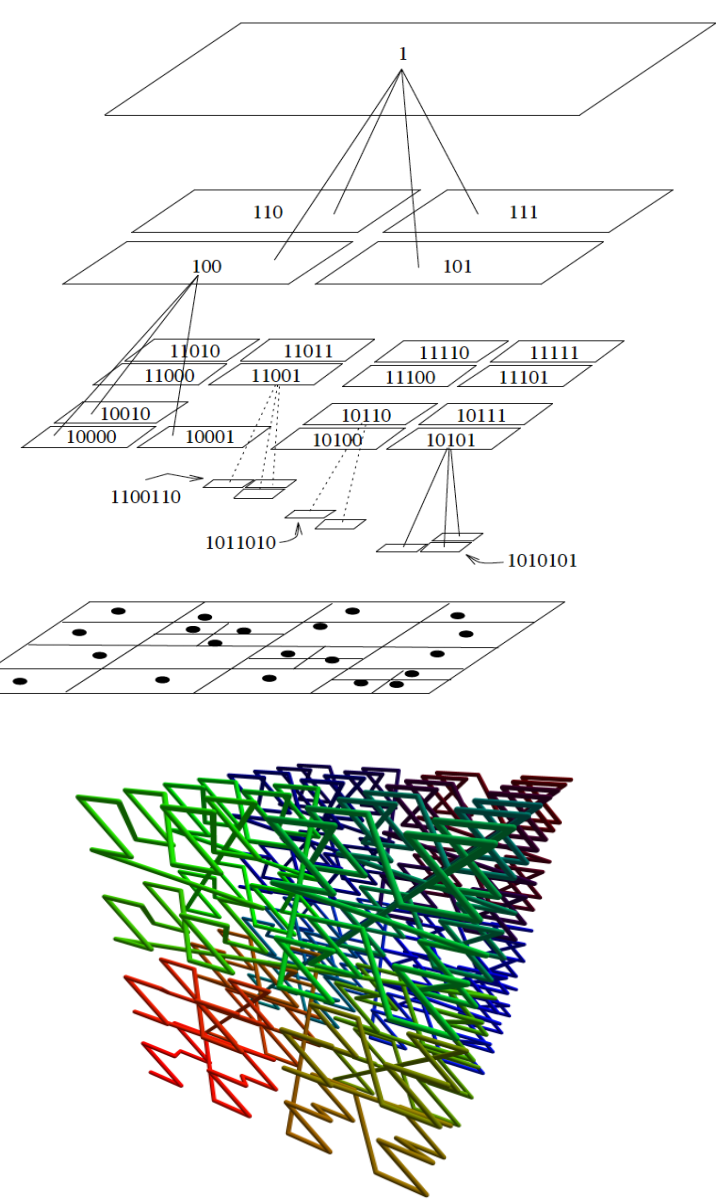


\section{How does FleCSI work?}

- Low-Level (FleCSI Core Capability)

- FleCSI provides a templated, low-level interface that can be specialized for a particular class of physics packages and application needs

- Specialization

- A specialization provides application developers with a high-level interface that is customized to their nomenclature and data structure requirements

- Task Abstraction (FleCSI Runtime Abstraction)

- Using the FleCSI task abstraction layer and some compile-time techniques, the application developer is given a programming system that is transparently distributed-memory parallel

- Kernel Abstraction (FleCSI Runtime Abstraction)

- Using the FleCSI kernel abstraction layer with compile-time techniques, application developers are given a programming system that is transparently fine-grained, data-parallel

\section{- Data Abstraction (FleCSI Core + Runtime)}

- FleCSI provides a data model that integrates with the task and kernel abstractions to provide easy registration and access to various data types with automatic dependency tracking 


\section{Where does FleCSI sit in the software stack?}

Provides high-level, domainspecific

interface that requires syntax and semantics not available in $\mathrm{C}++$

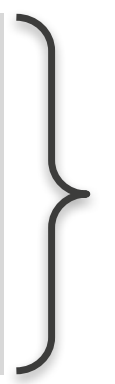

Data \& Execution Model Implementations

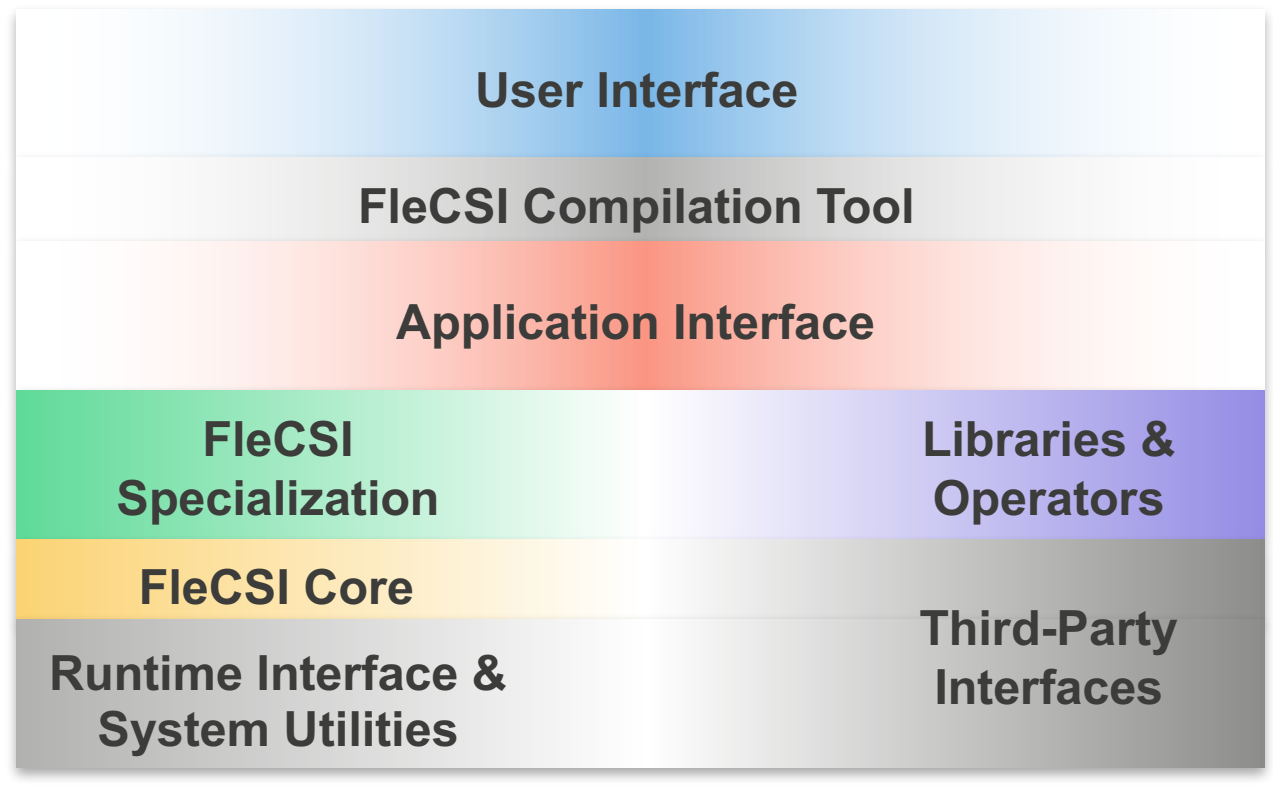




\section{contributions to FleCSI...}

Many people have made significant

Nick Moss (CCS-7)

Topology types, sparse data, design

Marc Charest (XCP-1)

Mesh specialization, data structures, design

Irina Demeshko (CCS-7)

Legion backend, MPI/Legion interoperability, design

Li-Ta (Ollie) Lo (CCS-7)

Partitioning interface, design

John Wohlbier (formerly CCS-2)

Mesh specialization, I/O

Josh Payne (CCS-7)

Execution model, design

Christoph Junghans (CCS-7)

Build system, continuous integration, Docker packaging Gary Dilts (CCS-2)

Tree specialization, data structures, design
Christopher Malone (CCS-2)

Remap implementation, design

David Daniel (CCS-7)

Requirements, design

Nathaniel Morgan (XCP-8)

Requirements

Vince Chiravalle (A-2)

Requirements

Joe Schmidt (XTD-NTA)

Requirements

Chris Sewell (CCS-7)

Requirements, design

Rao Garimella (T-7)

Requirements, design

Wes Even (CCS-2)

Requirements, design

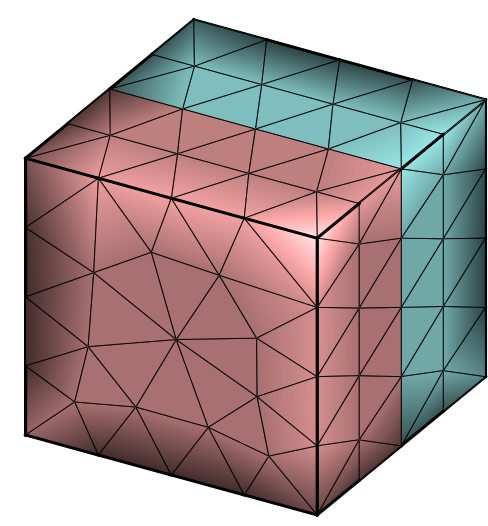

Multiblock unstructured meshes

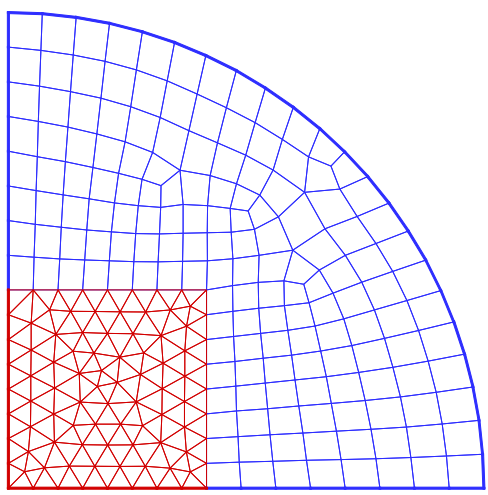

Mixed triangles and quads 


\section{Separation of Concerns}




\section{Our project structure separates roles and concerns...}

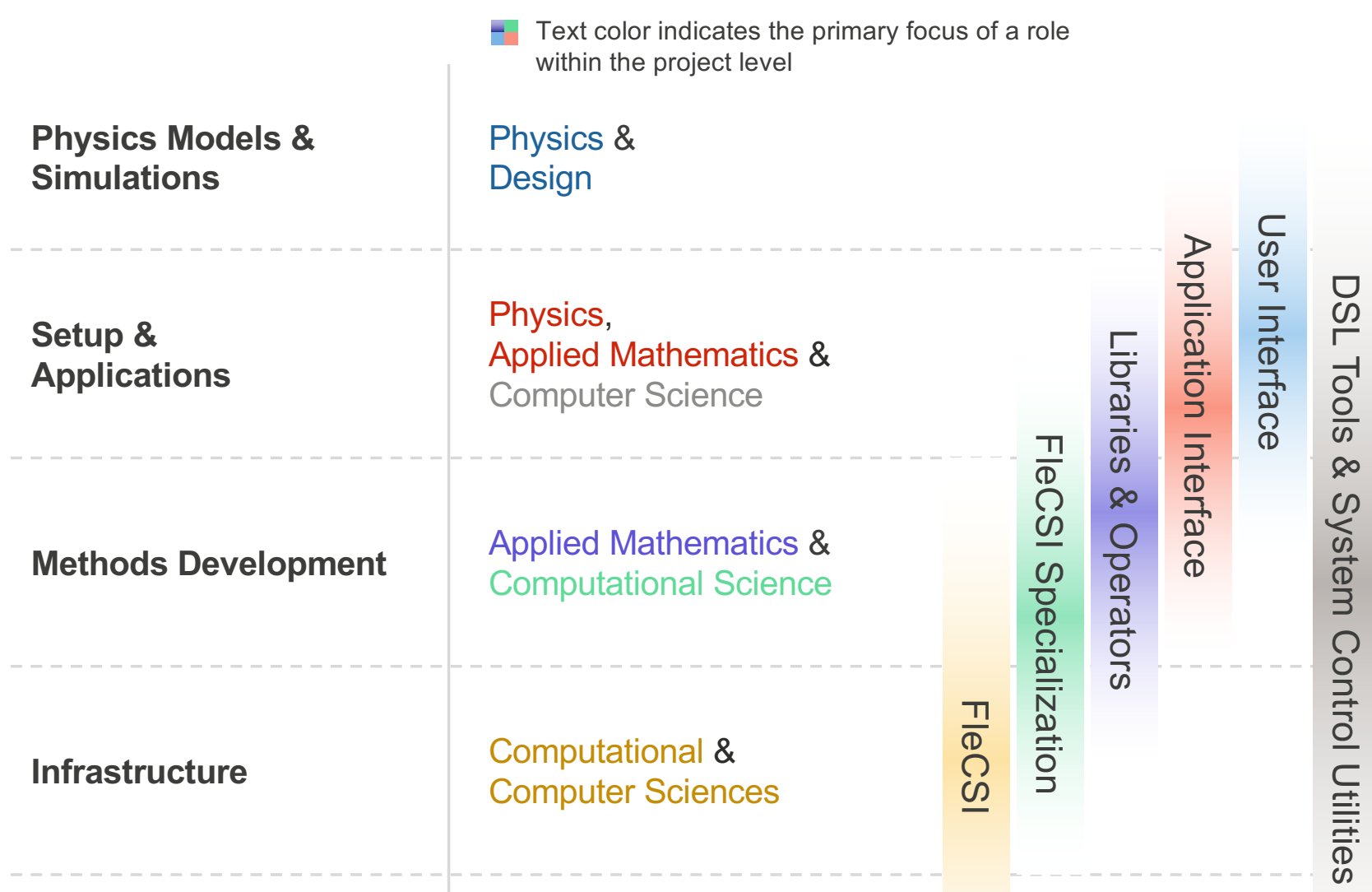




\section{FleCSI Control/Execution Model}


The FleCSI execution/control model is a reflection of how we (LANL) think about multi-physics...

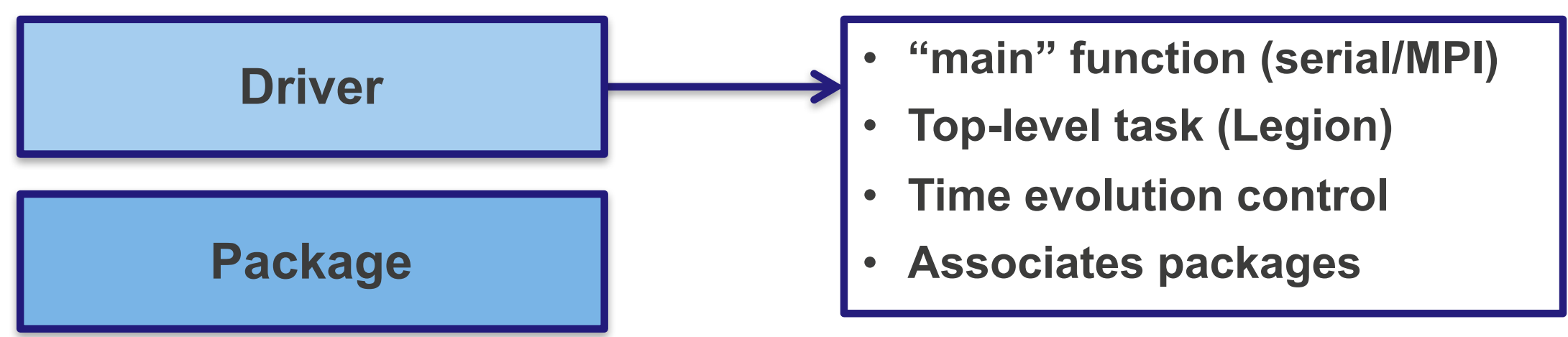

Task

Kernel 
The FleCSI execution/control model is a reflection of how we (LANL) think about multi-physics...

\section{Driver}

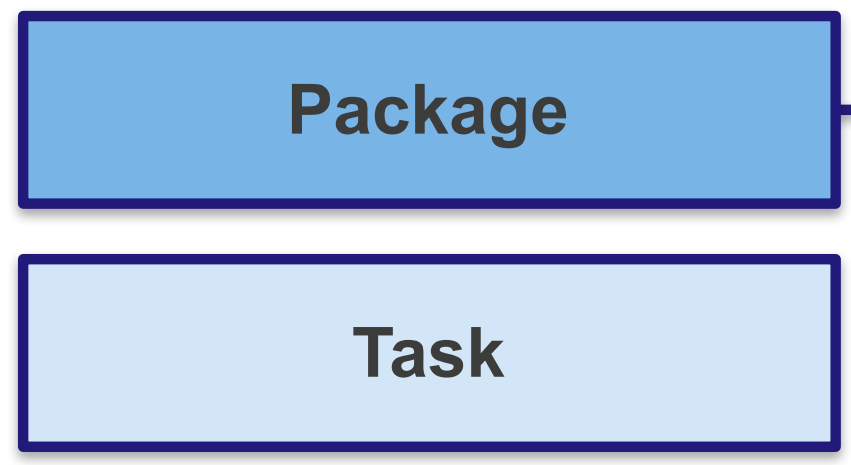

- Namespace for managing or associating tasks

Kernel 


\section{The FleCSI execution/control model is a reflection of how we (LANL) think about multi-physics...}

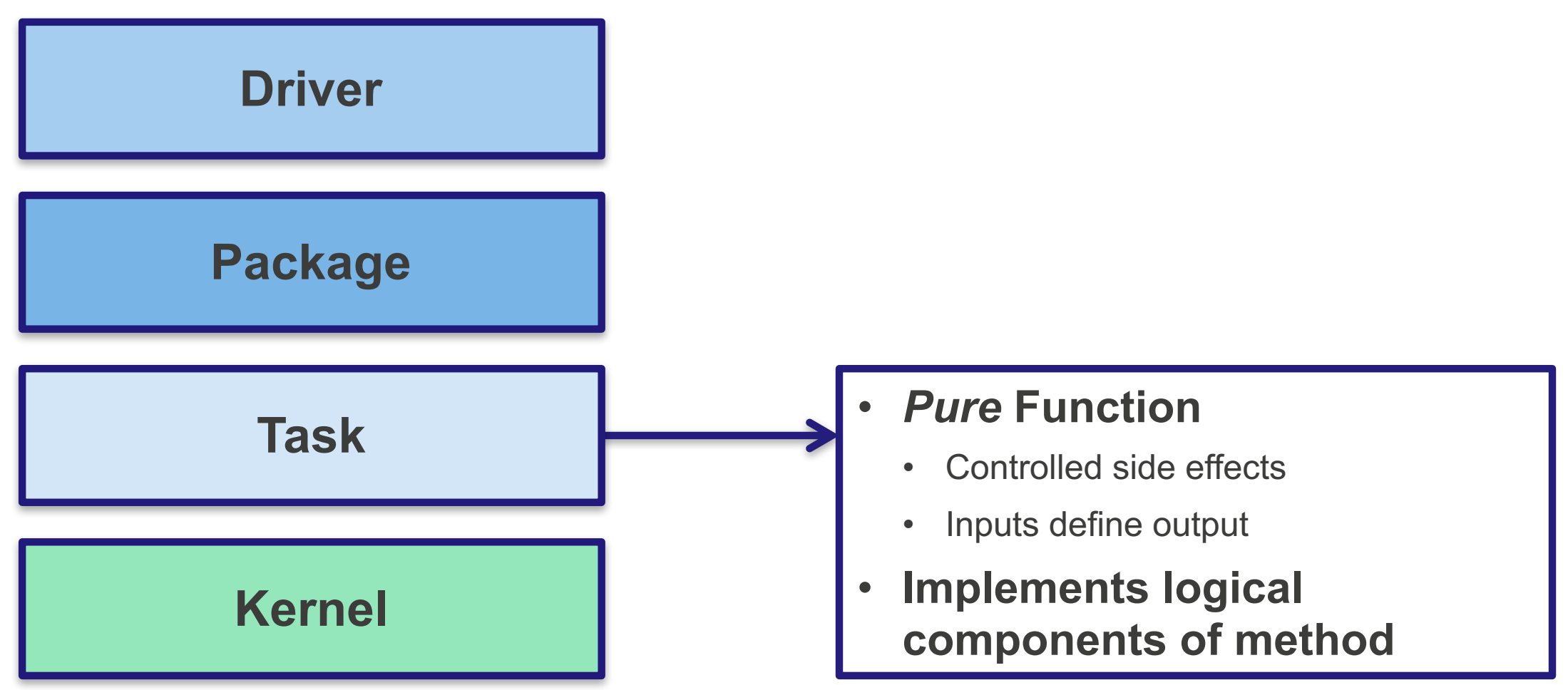




\section{The FleCSI execution/control model is a reflection of how we (LANL) think about multi-physics...}

\section{Driver}

\section{Package}

Task

Kernel

- Fine-grained, data-parallel functions, e.g., foreach

- Use on-node runtimes: OpenMP, Thrust, $\mathrm{C}++17$ 


\section{FleCSI Data Model Interface}




\section{What is the FleCSI data interface?}

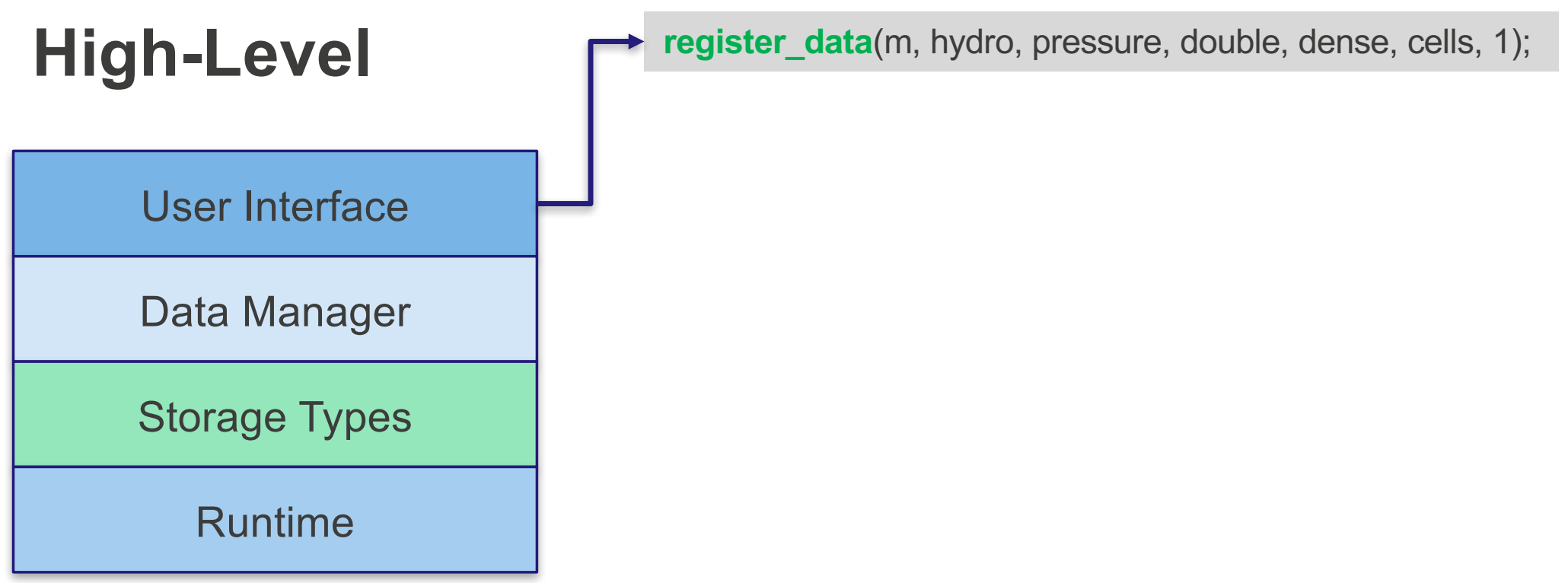




\section{What is the FleCSI data interface?}

\section{High-Level}

\begin{tabular}{|c|}
\hline User Interface \\
\hline Data Manager \\
\hline Storage Types \\
\hline Runtime \\
\hline
\end{tabular}

register_data(m, hydro, pressure, double, dense, cells, 1);

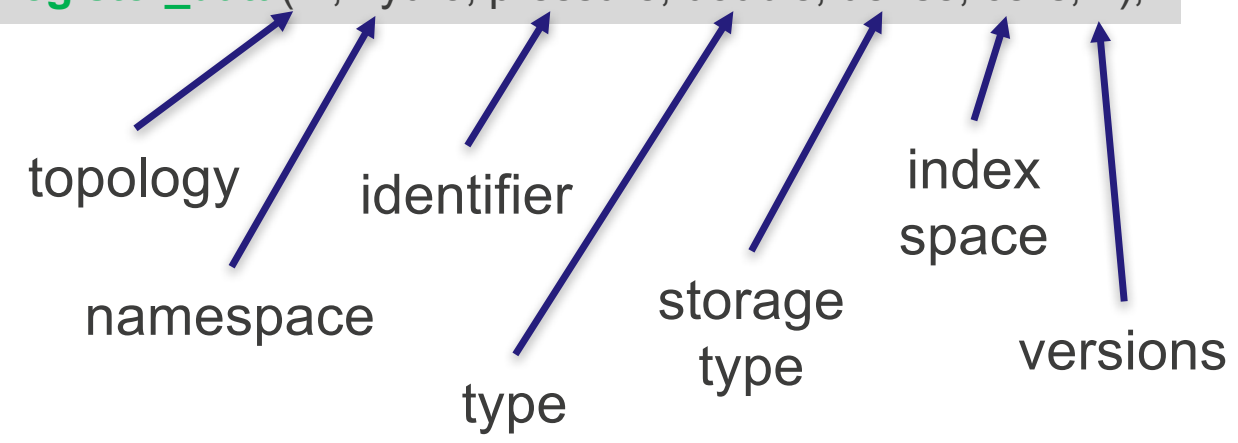




\section{What is the FleCSI data interface?}

\section{Direct Interface}

\begin{tabular}{|c|}
\hline User Interface \\
\hline Data Manager \\
\hline Storage Types \\
\hline Runtime \\
\hline
\end{tabular}

template<size_t ST, typename T, size_t NS, typename ... Args> decltype(auto) register_data( data_client_t \& data_client, const const_string_t \& key, size_t versions $=1$,

Args \&\& ... args) 


\section{What is the FleCSI data interface?}

\section{Direct Interface}

\begin{tabular}{|c|}
\hline User Interface \\
\hline Data Manager \\
\hline Storage Types \\
\hline Runtime \\
\hline
\end{tabular}

template<size_t ST, typename T, size_t NS, typename ... Args> decltype(auto) register_data( data_client_t \& data_client, $\longleftarrow$ topology const const_string_t \& key, size_t versions $=1$, Args \&\& ... args) 


\section{What is the FleCSI data interface?}

\section{Low-Level}

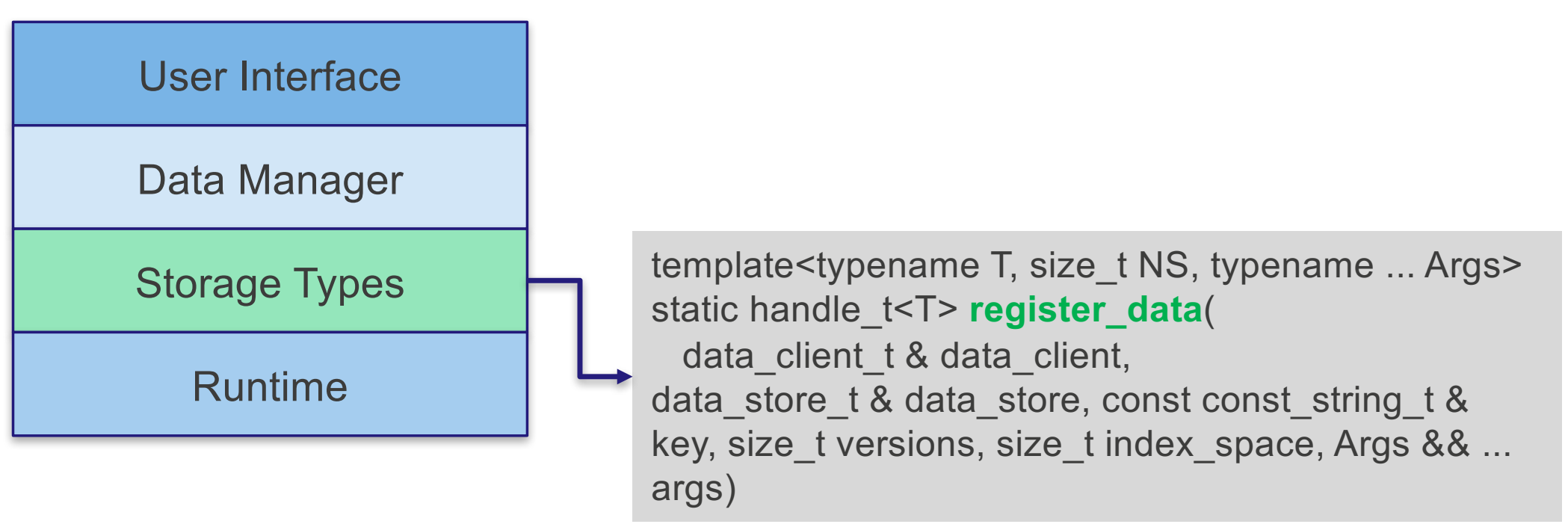




\section{What is the FleCSI data interface?}

\section{Low-Level}

\begin{tabular}{|c|}
\hline User Interface \\
\hline Data Manager \\
\hline Storage Types \\
\hline Runtime \\
\hline
\end{tabular}

Mapped to specific storage type

template<typename T, size_t NS, typename ... Args $>$ static handle_t $<T>$ register_data( data_client_t \& data_client, data_store_t \& data_store, const const_string_t \& key, size_t versions, size_t index_space, Args \& \& ... args) 


\section{What is the FleCSI data interface?}

\section{Backend}

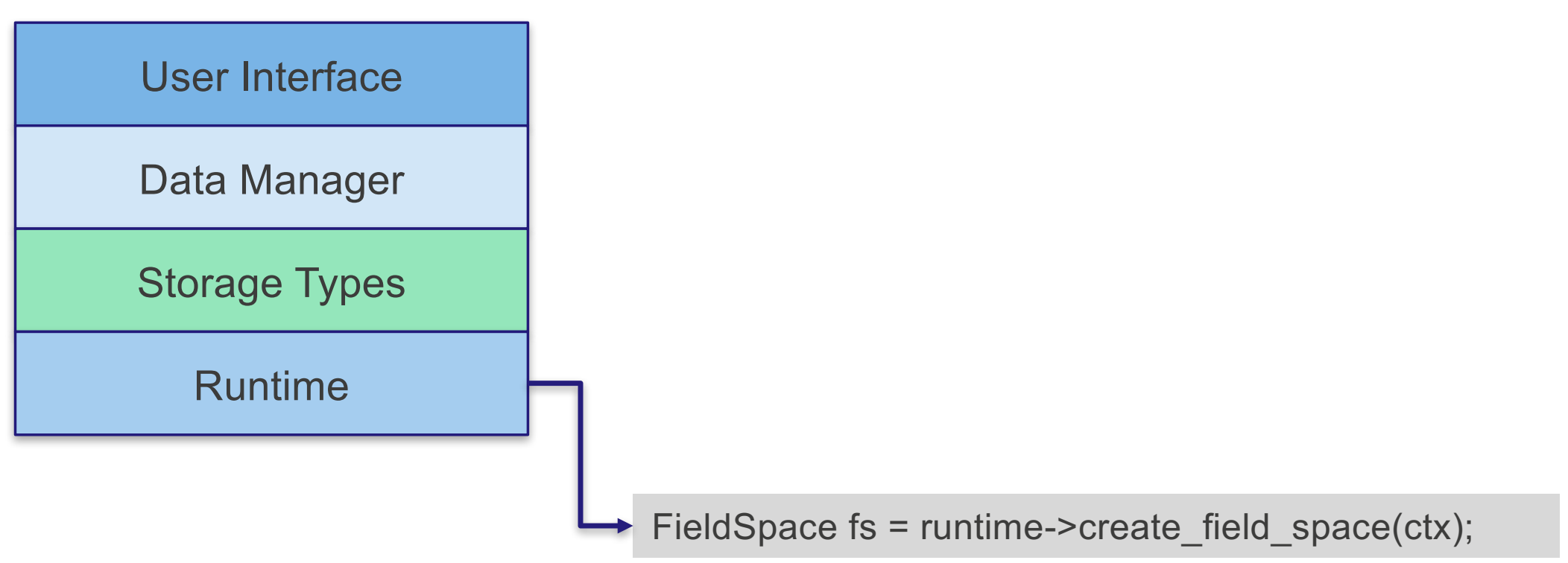




\section{What is the FleCSI data interface?}

\section{Backend}

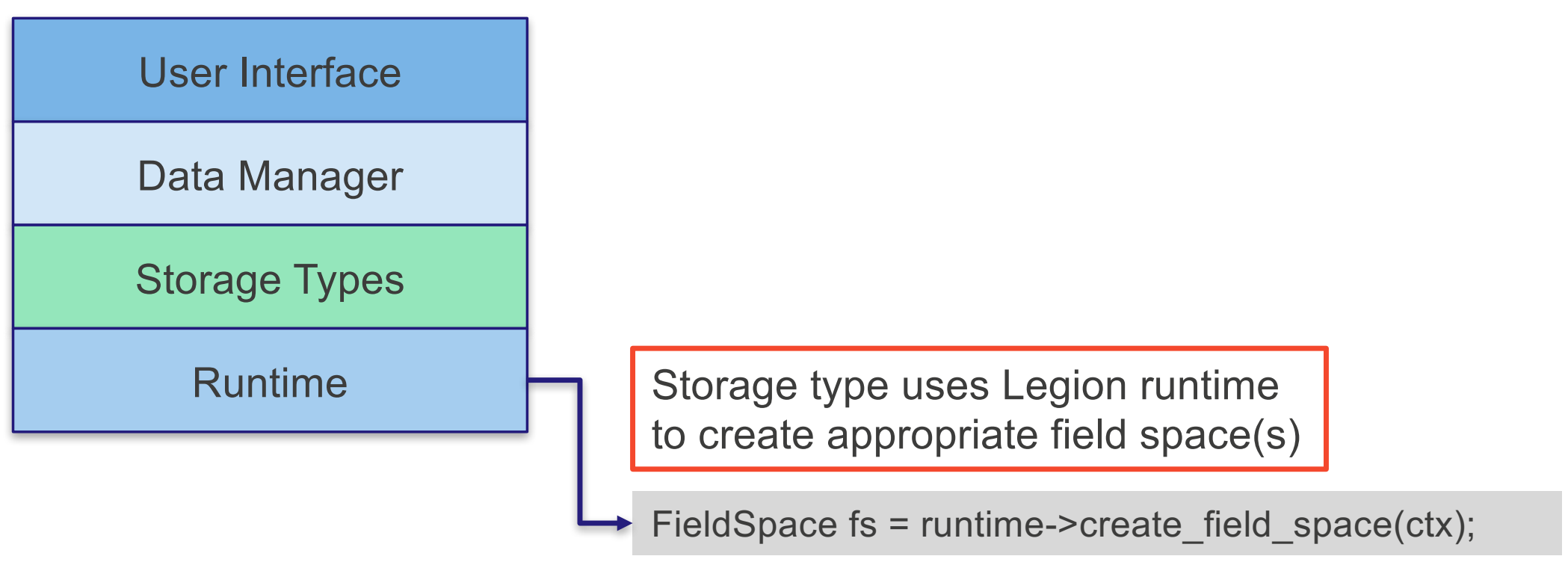




\section{What storage types do we support?}

- Dense: One dimensional, contiguous array

- Use Case: Physics state data

- Global: Single data instance (there's only one...)

- Use Case: Simulation state data

- Local: One dimensional, contiguous array

- Use Case: Scratch data

- Sparse: Dense index space, sparse population

- Use Case: Material data, execution-dependent data, sparse matrices

- Tuple: Combination of other storage types

- Use Case: Provide struct-like support for cleaner task definitions 


\section{Sparse Data Storage Type}




\section{Managing sparse data representations presents some challenges...}

Initial Distribution

$\square$ material 1

material 2

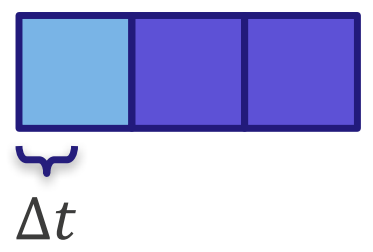

Matrix Representation

$$
\begin{array}{lll} 
& \mathrm{m} 1 & \mathrm{~m} 2 \\
\mathrm{c0} & \mathrm{v} 1 & \\
\mathrm{c1} & & \mathrm{v} 2
\end{array}
$$

\begin{tabular}{|c|c|c|c|c|}
\hline offsets & 0 & 1 & 2 & 3 \\
\hline values & $\mathrm{v} 1$ & v2 & v3 & \\
\hline indices & 0 & 1 & 1 & \\
\hline
\end{tabular}

Compressed Storage

c2

v3 


\section{Managing sparse data representations presents some challenges...}

Evolved Distribution

$\square$ material 1

material 2

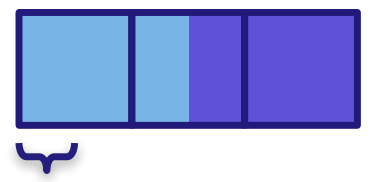

$\Delta t \longrightarrow$ linear advection
Matrix Representation

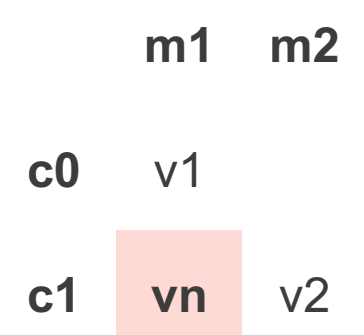

c2

v3

Compressed Storage

offsets

\begin{tabular}{|l|l|l|l|}
\hline 0 & 1 & 3 & 4 \\
\hline
\end{tabular}

values

\begin{tabular}{|l|l|l|l|}
\hline v1 & vn & v2 & v3 \\
\hline
\end{tabular}

indices

\begin{tabular}{|l|l|l|l|}
\hline 0 & $\mathbf{0}$ & 1 & 1 \\
\hline
\end{tabular}

Mutated Structure!!! 


\section{How does FleCSI handle sparse data?}

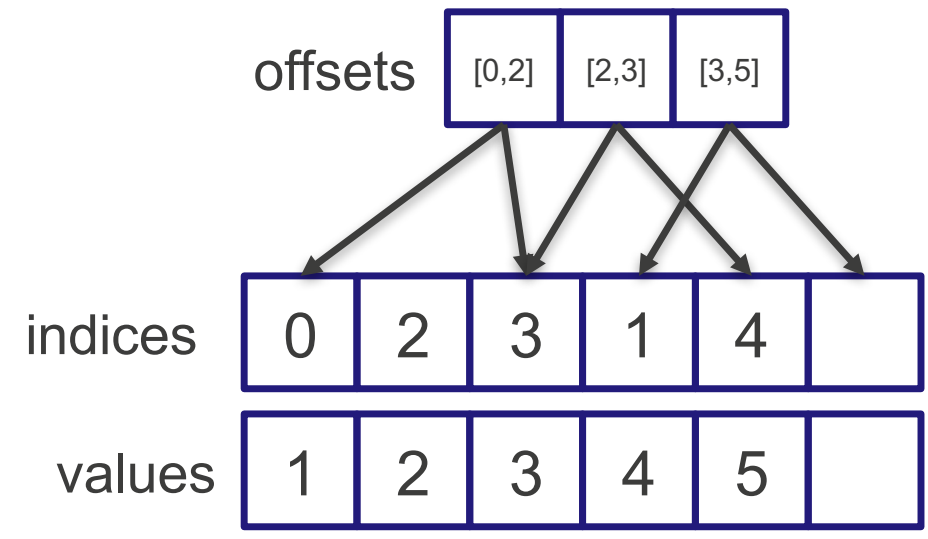

$$
A=\left[\begin{array}{lllll}
1 & 0 & 2 & 0 & 0 \\
0 & 0 & 0 & 3 & 0 \\
0 & 4 & 0 & 0 & 5
\end{array}\right]
$$




\section{How does FleCSI handle sparse data?}

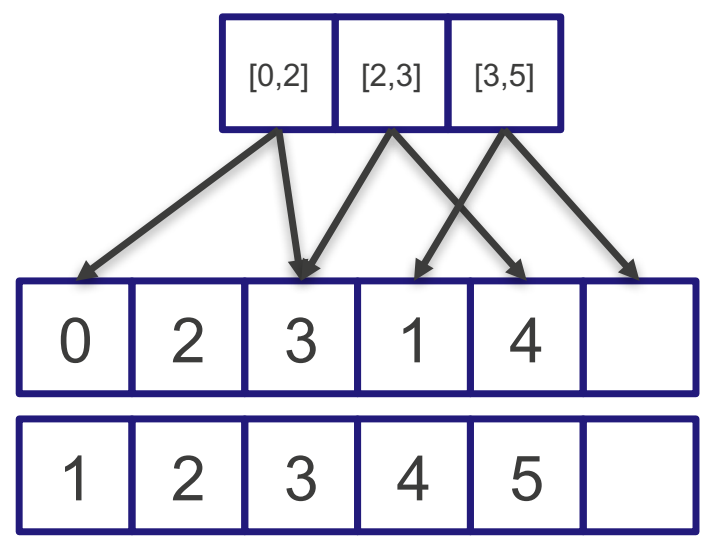

$$
A=\left[\begin{array}{lllll}
1 & 0 & 2 & 0 & 0 \\
0 & 0 & 0 & 3 & 0 \\
0 & 4 & 0 & 0 & 5
\end{array}\right]
$$

Code block to mutate sparse structure

$$
\begin{aligned}
& \{ \\
& m=\text { get_mutator }(\mathrm{A}, 3) \\
& \mathrm{m}[0][1]=6 \\
& \} / / \text { scope }
\end{aligned}
$$




\section{How does FleCSI handle sparse data?}

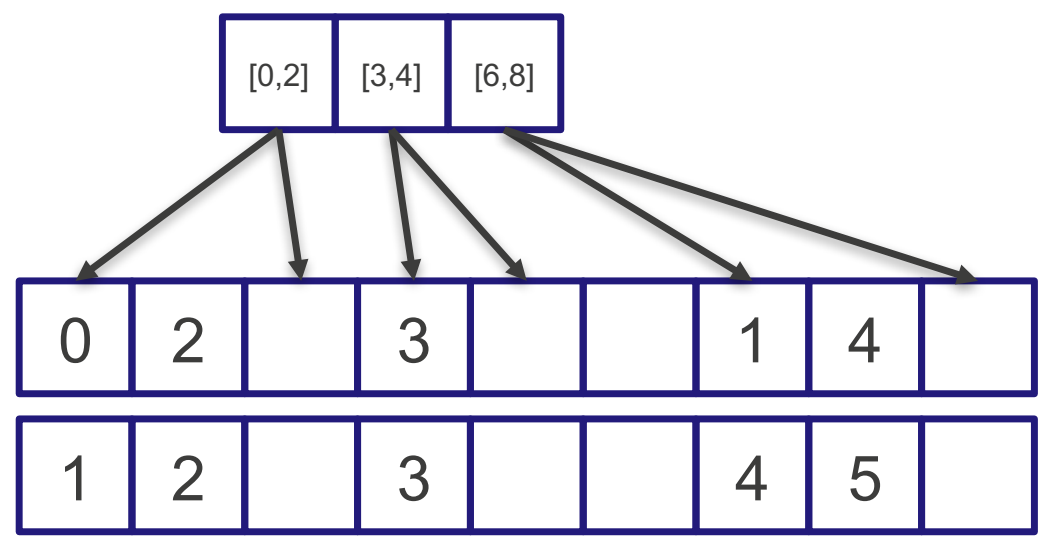

$$
A=\left[\begin{array}{lllll}
1 & 0 & 2 & 0 & 0 \\
0 & 0 & 0 & 3 & 0 \\
0 & 4 & 0 & 0 & 5
\end{array}\right]
$$

Constructor inserts space for new values

\{

$\mathrm{m}=$ get_mutator(A, 3); $\mathrm{m}[0][1]=6$;

\}$/ /$ scope

Generic Compressed Sparse Matrix Insertion: Algorithms and Implementations in MTL4 and FEniCS POOSC '09 Proceedings of the 8th workshop on Parallel/High-Performance Object-Oriented Scientific Computing 


\section{How does FleCSI handle sparse data?}

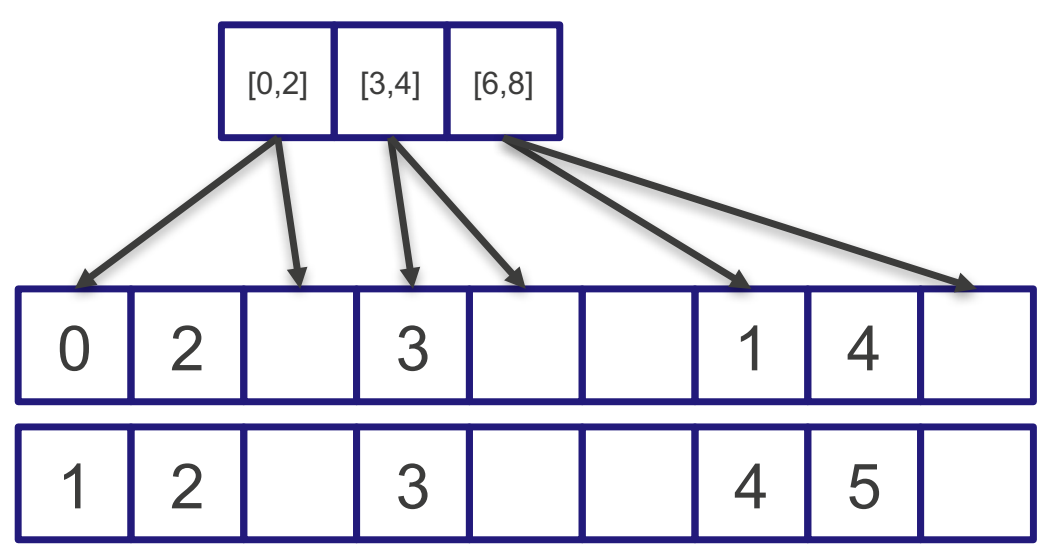

Constructor inserts space for new values

$$
A=\left[\begin{array}{lllll}
1 & 0 & 2 & 0 & 0 \\
0 & 0 & 0 & 3 & 0 \\
0 & 4 & 0 & 0 & 5
\end{array}\right]
$$

User specifies maximum total slots

\{

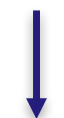

$\mathrm{m}=$ get_mutator(A, 3); $\mathrm{m}[0][1]=6$;

\}// scope 


\section{How does FleCSI handle sparse data?}

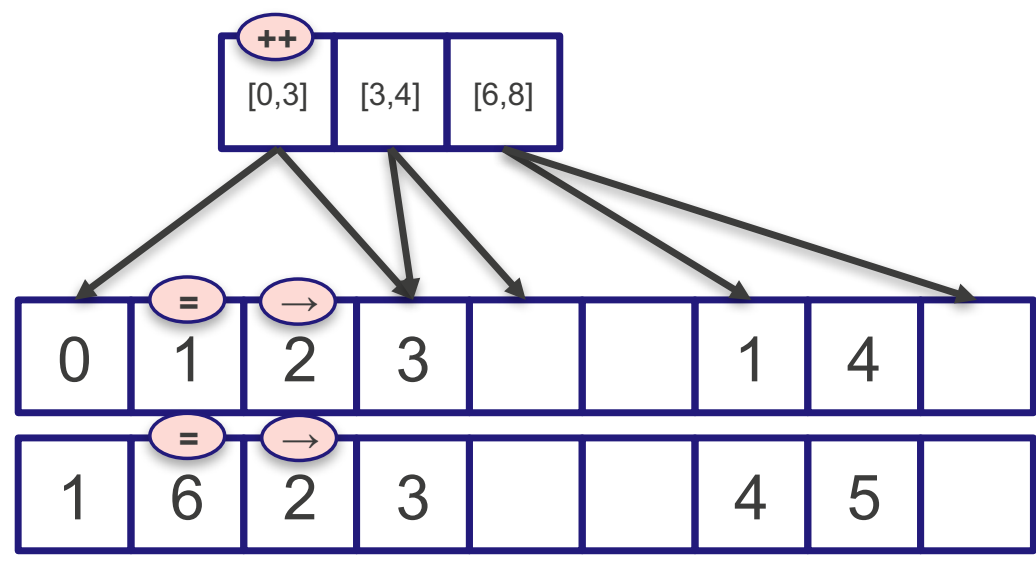

$$
A=\left[\begin{array}{ccccc}
1 & 6 & 2 & 0 & 0 \\
0 & 0 & 0 & 3 & 0 \\
0 & 4 & 0 & 0 & 5
\end{array}\right]
$$

Intuitive interface to set non-zeros

$$
\{
$$

$\mathrm{m}=$ get_mutator(A, 3); $\mathrm{m}[0][1]=6$;

\}$/ /$ scope

Generic Compressed Sparse Matrix Insertion: Algorithms and Implementations in MTL4 and FEniCS POOSC '09 Proceedings of the 8th workshop on Parallel/High-Performance Object-Oriented Scientific Computing 


\section{How does FleCSI handle sparse data?}

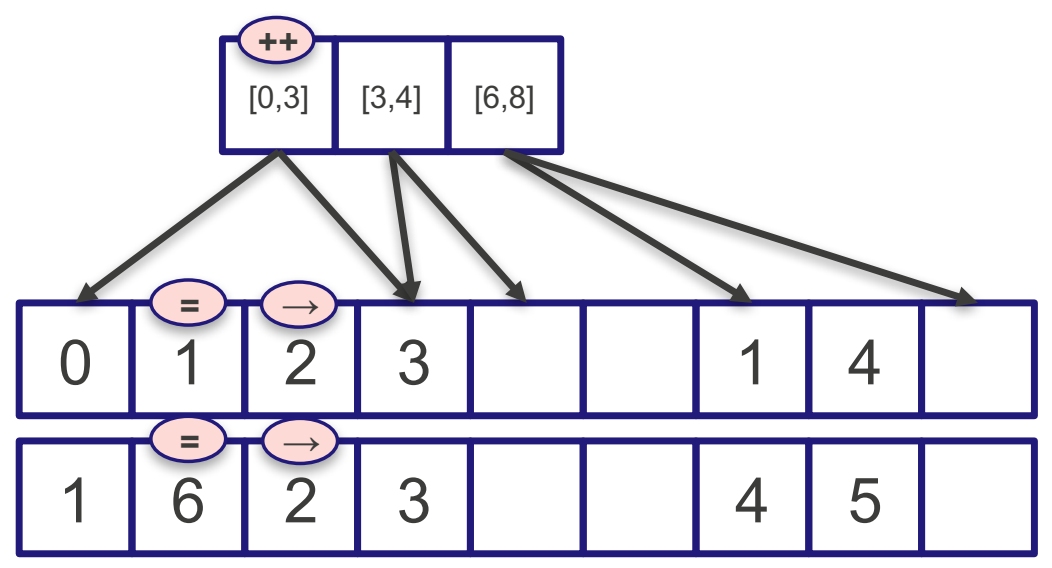

$$
A=\left[\begin{array}{ccccc}
1 & 6 & 2 & 0 & 0 \\
0 & 0 & 0 & 3 & 0 \\
0 & 4 & 0 & 0 & 5
\end{array}\right]
$$

\{

Intuitive interface to set non-zeros $\longrightarrow \quad \mathrm{m}[0][1]=6$;

$\mathrm{m}=$ get_mutator(A, 3);

(logically, $m$ has a $5 \times 3$ dense structure like $A$ )

\}// scope

Generic Compressed Sparse Matrix Insertion: Algorithms and Implementations in MTL4 and FEniCS

POOSC '09 Proceedings of the 8th workshop on Parallel/High-Performance Object-Oriented Scientific Computing 


\section{How does FleCSI handle sparse data?}

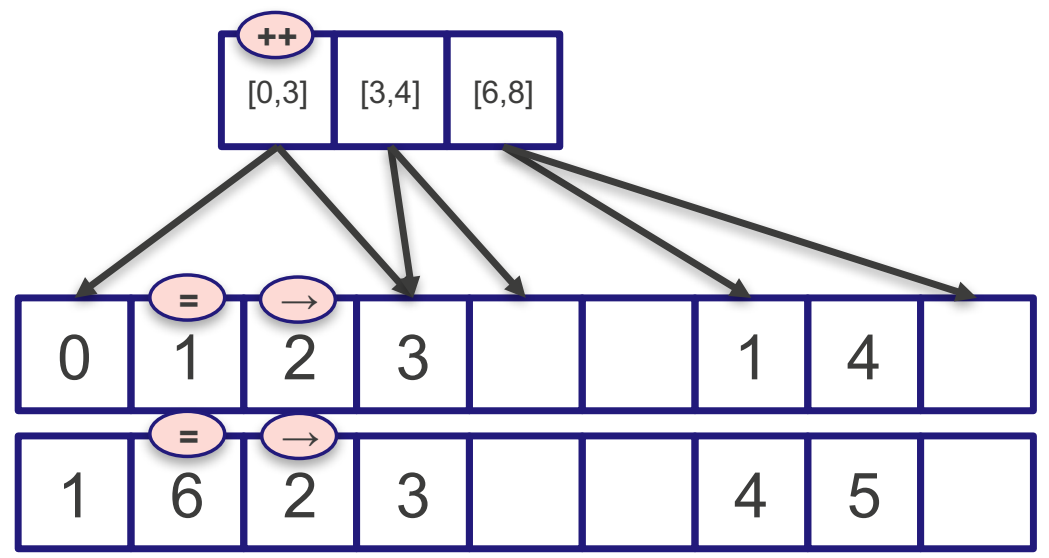

Column order is preserved, single-slot shift, only slot end is incremented

Intuitive interface to set non-zeros

$$
A=\left[\begin{array}{ccccc}
1 & 6 & 2 & 0 & 0 \\
0 & 0 & 0 & 3 & 0 \\
0 & 4 & 0 & 0 & 5
\end{array}\right]
$$

\{

$\mathrm{m}=$ get_mutator(A, 3); $\mathrm{m}[0][1]=6$;

\}// scope 


\section{How does FleCSI handle sparse data?}

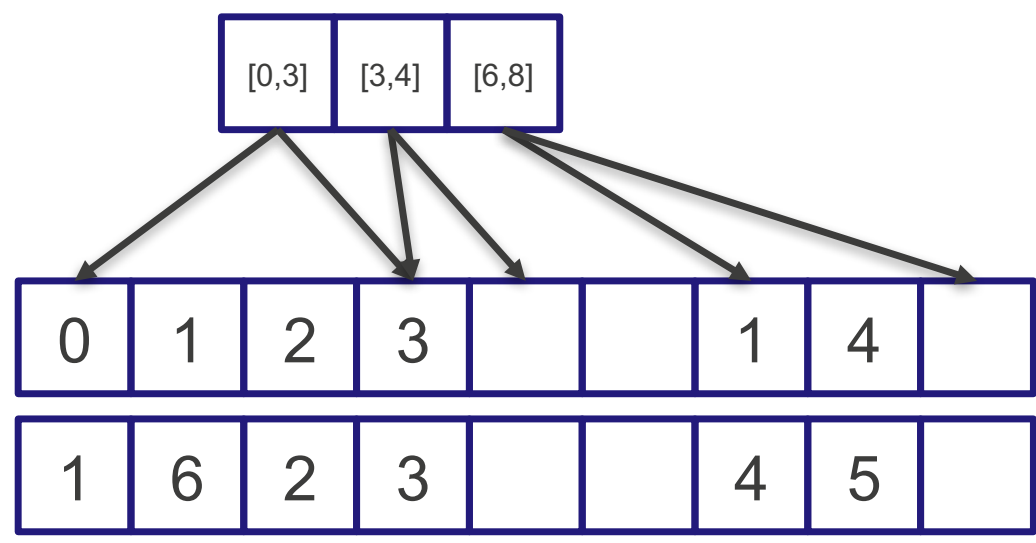

$$
A=\left[\begin{array}{lllll}
1 & 6 & 2 & 0 & 0 \\
0 & 0 & 0 & 3 & 0 \\
0 & 4 & 0 & 0 & 5
\end{array}\right]
$$

What if we need more than 3 non-zeros?

\{

$\mathrm{m}=$ get_mutator(A, 3); $\mathrm{m}[0][1]=6$;

\}$/ /$ scope

Generic Compressed Sparse Matrix Insertion: Algorithms and Implementations in MTL4 and FEniCS POOSC '09 Proceedings of the 8th workshop on Parallel/High-Performance Object-Oriented Scientific Computing 


\section{How does FleCSI handle sparse data?}

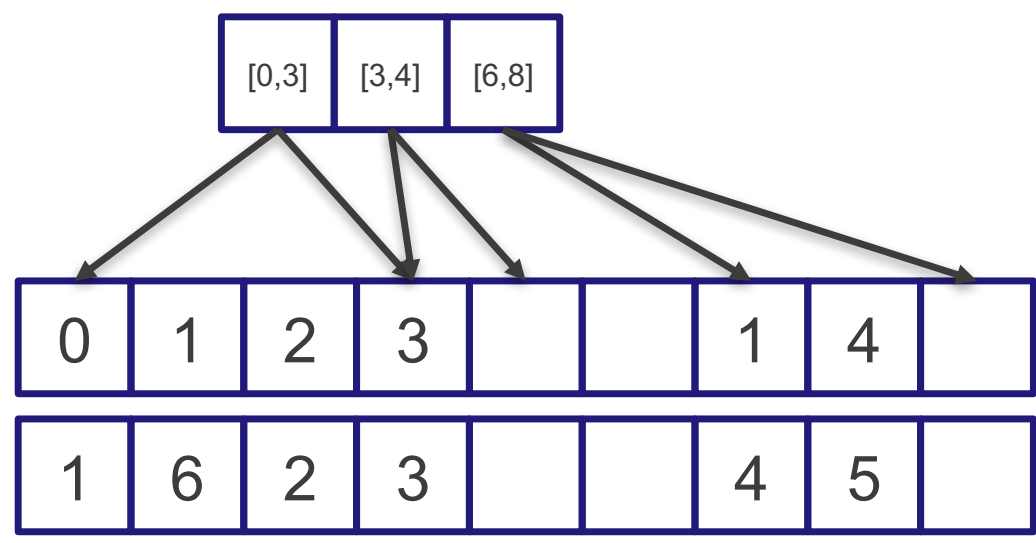

$$
A=\left[\begin{array}{lllll}
1 & 6 & 2 & (7) & 0 \\
0 & 0 & 0 & 3 & 0 \\
0 & 4 & 0 & 0 & 5
\end{array}\right]
$$

What if we need more than 3 non-zeros?

$$
\{
$$

$\mathrm{m}=$ get_mutator(A, 3); $\mathrm{m}[0][3]=7$;

\}$/ /$ scope

Generic Compressed Sparse Matrix Insertion: Algorithms and Implementations in MTL4 and FEniCS POOSC '09 Proceedings of the 8th workshop on Parallel/High-Performance Object-Oriented Scientific Computing 


\section{How does FleCSI handle sparse data?}

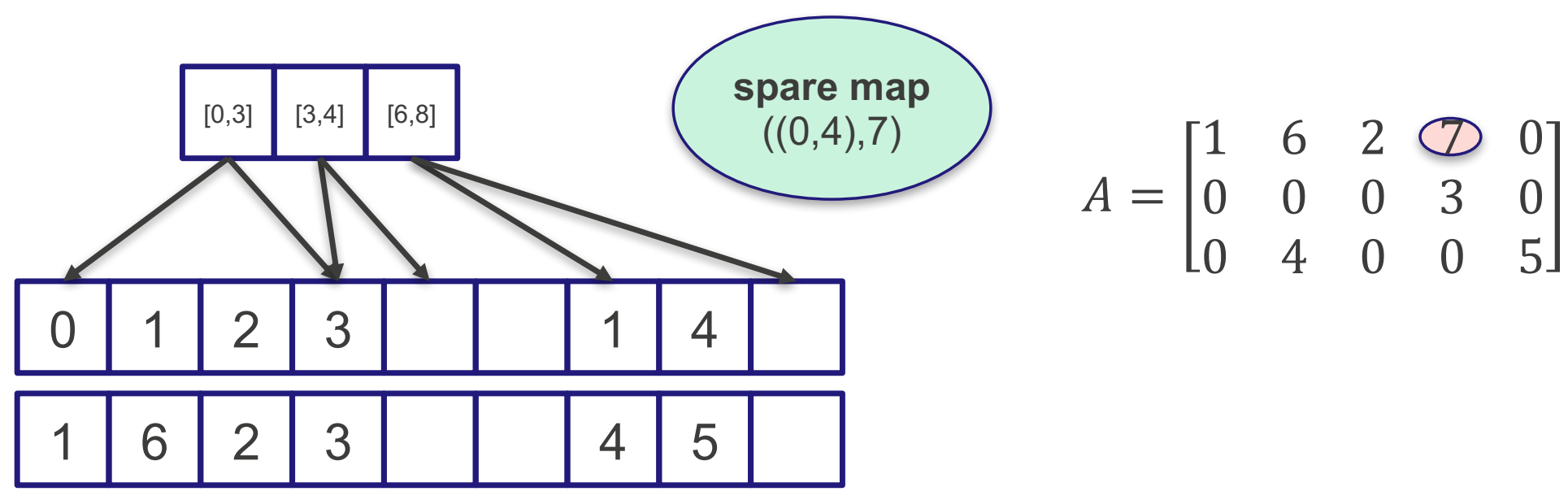

What if we need more than 3 non-zeros $? \longrightarrow \quad \mathrm{m}[0][3]=7 ;$
\}$/ /$ scope

\{

POOSC '09 Proceedings of the 8th workshop on Parallel/High-Performance Object-Oriented Scientific Computing 


\section{How does FleCSI handle sparse data?}

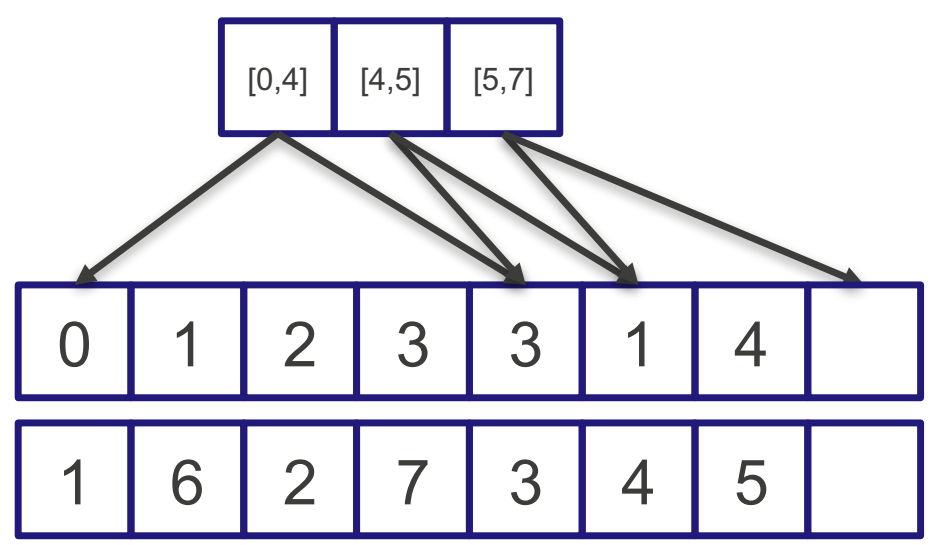

$$
A=\left[\begin{array}{lllll}
1 & 6 & 2 & 7 & 0 \\
0 & 0 & 0 & 3 & 0 \\
0 & 4 & 0 & 0 & 5
\end{array}\right]
$$

Destructor recompresses data $\longrightarrow \quad$ \}// scope 


\section{How does FleCSI handle sparse data?}

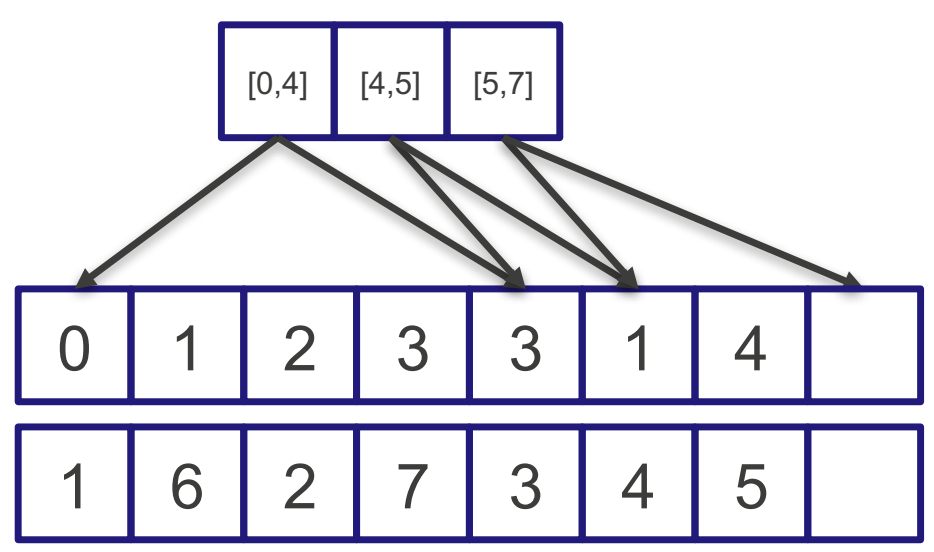

$$
A=\left[\begin{array}{lllll}
1 & 6 & 2 & 7 & 0 \\
0 & 0 & 0 & 3 & 0 \\
0 & 4 & 0 & 0 & 5
\end{array}\right]
$$

The time complexity for inserting $n$ non-zeros is $\mathrm{O}(n)$ for direct insertion and $\mathrm{O}(n \log (n))$ for indirect insertion. 


\section{How does FleCSI handle sparse data?}

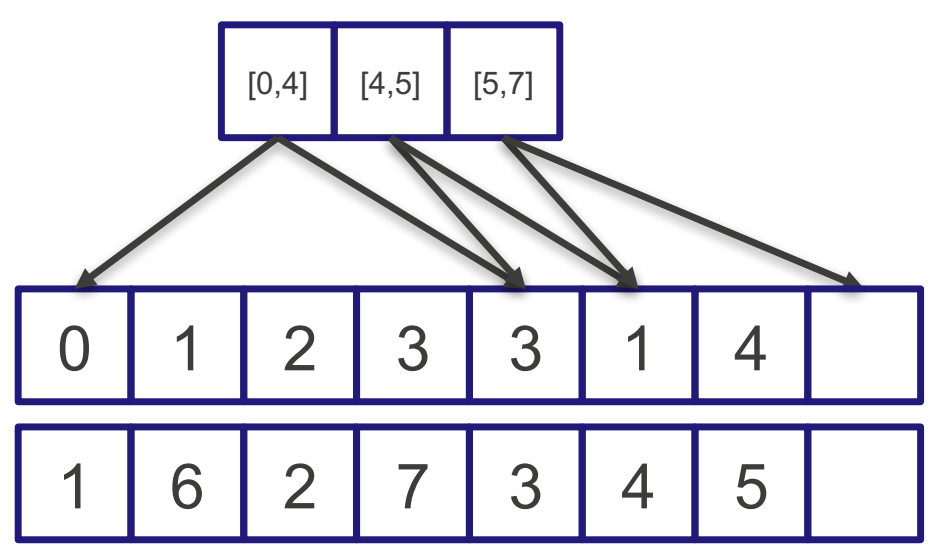

$$
A=\left[\begin{array}{lllll}
1 & 6 & 2 & 7 & 0 \\
0 & 0 & 0 & 3 & 0 \\
0 & 4 & 0 & 0 & 5
\end{array}\right]
$$

The memory complexity depends on the application, but can be quite efficient if good estimates are known. 


\section{How does FleCSI handle sparse data?}

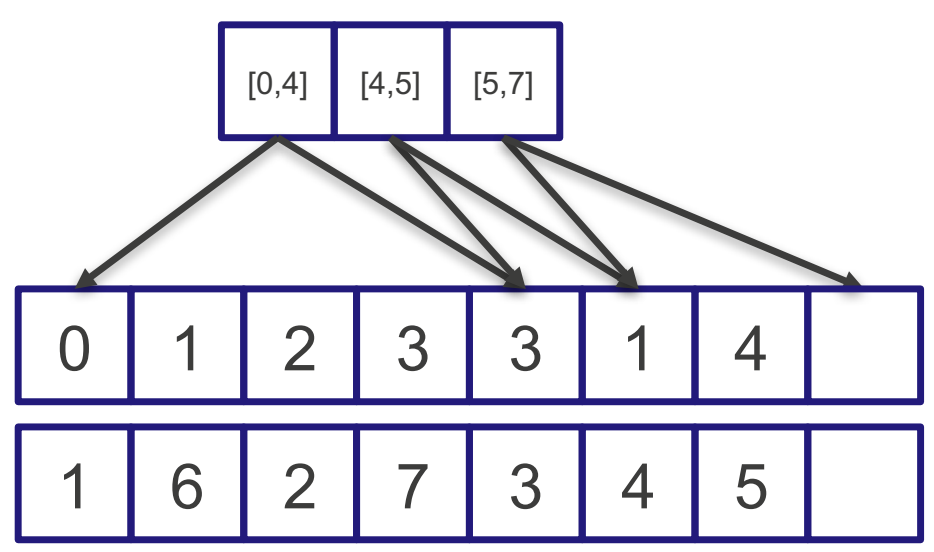

$$
A=\left[\begin{array}{lllll}
1 & 6 & 2 & 7 & 0 \\
0 & 0 & 0 & 3 & 0 \\
0 & 4 & 0 & 0 & 5
\end{array}\right]
$$

Implementation will support ELL-like dense number of materials format, i.e., all rows have space for $m$ non-zeros 


\section{How does FleCSI handle sparse data?}

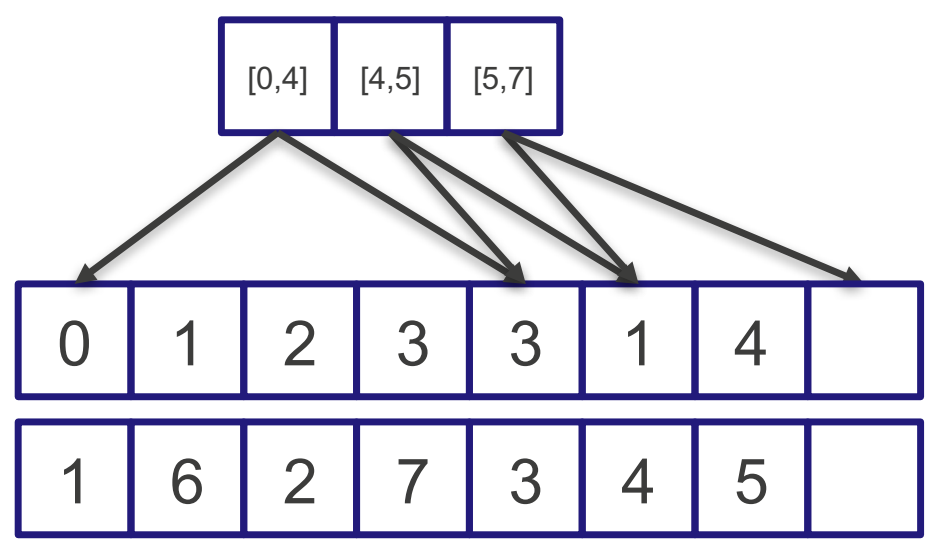

$$
A=\left[\begin{array}{lllll}
1 & 6 & 2 & 7 & 0 \\
0 & 0 & 0 & 3 & 0 \\
0 & 4 & 0 & 0 & 5
\end{array}\right]
$$

We will also support nested sparsity through the use of data handles. 


\section{How does FleCSI handle sparse data?}

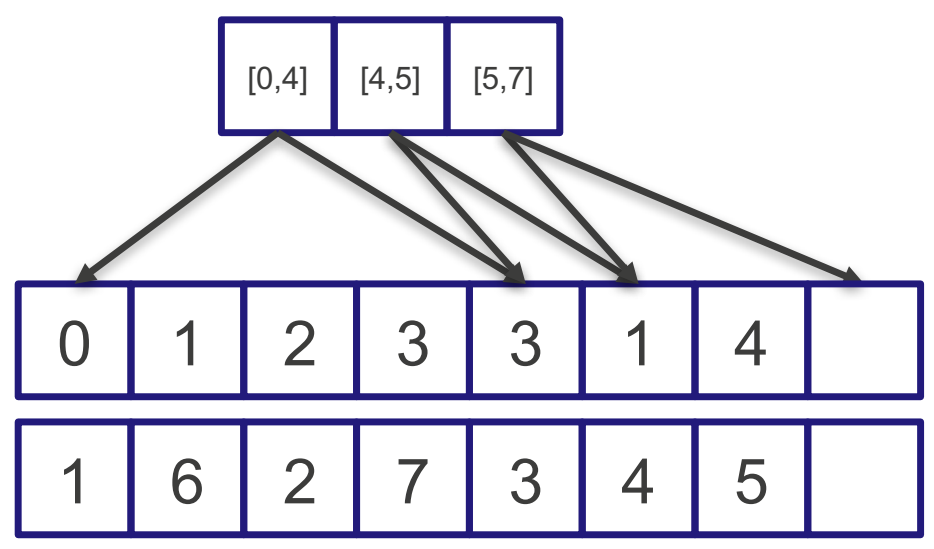

$$
A=\left[\begin{array}{lllll}
1 & 6 & 2 & 7 & 0 \\
0 & 0 & 0 & 3 & 0 \\
0 & 4 & 0 & 0 & 5
\end{array}\right]
$$

We will also support nested sparsity through the use of data handles. 


\section{FleCSI Execution Model Interface}




\section{What is the FleCSI execution interface?}

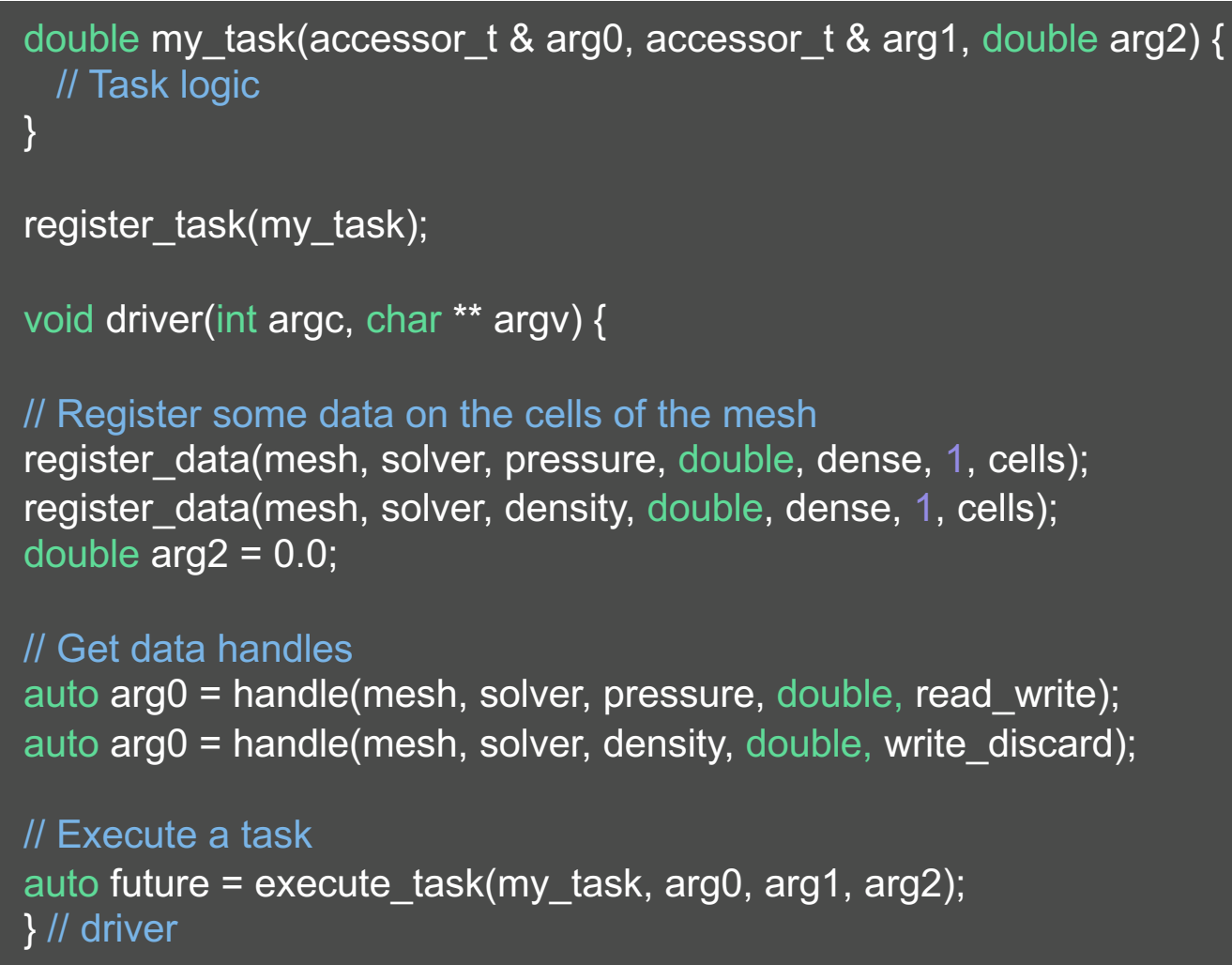




\section{Task Execution Flow}

execute_task(user_task, handle args...)

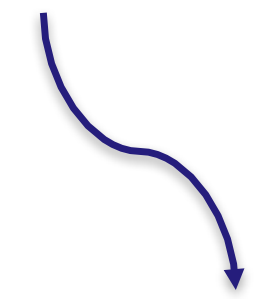

flecsi::execute_task (static processing)

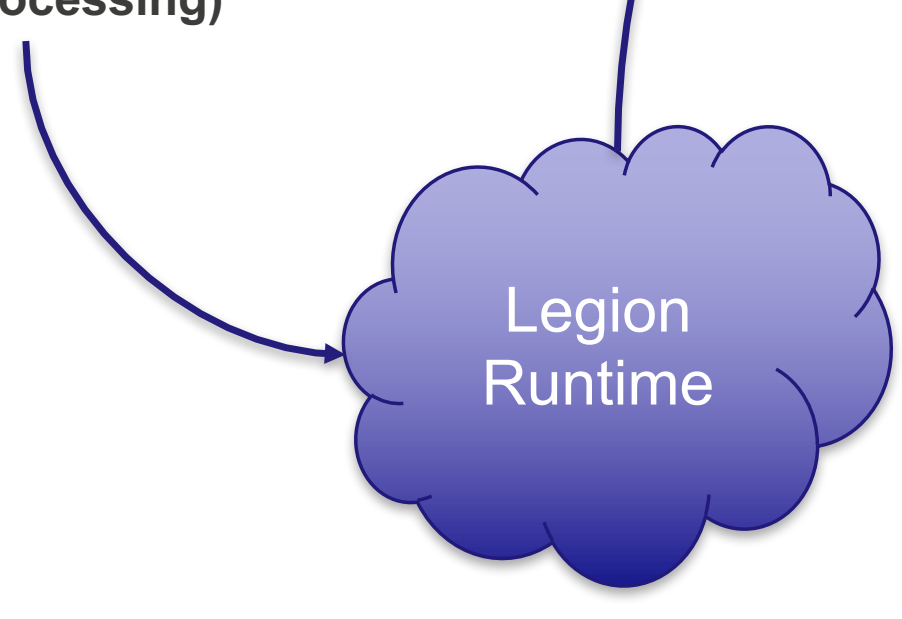

flecsi::task_wrapper

(static processing)

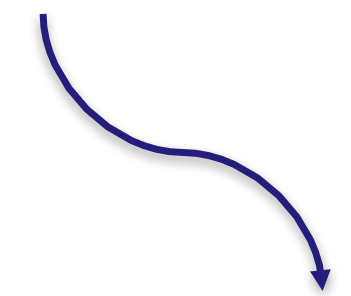

user_task(accessor args...) 


\section{Processing Data Handle Arguments}
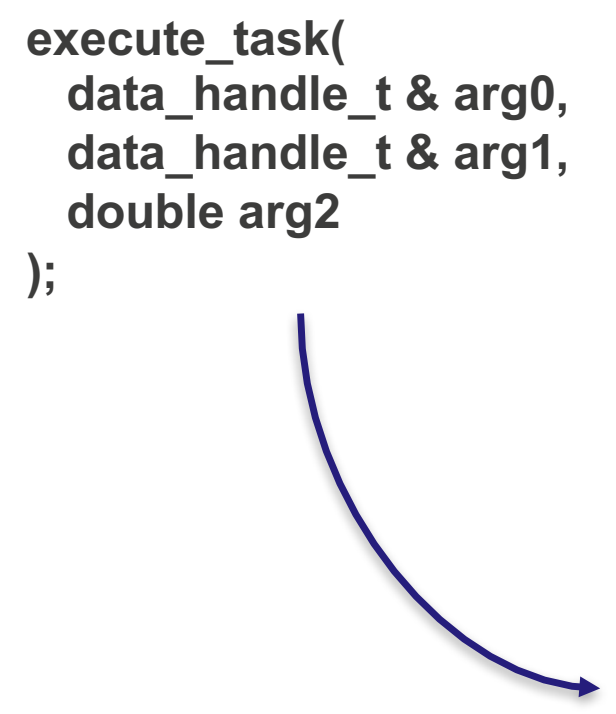

tuple_for_each(args, [\&](auto \& arg) \{

if(std::is_base_of<data_handle_t, decltype(arg)>::value) \{

launcher.add_region_requirement $\left({ }^{*}\right.$ values from handle * $\left./\right)$;

launcher.add_field $(/ *$ one or more field handles * $/)$;

\}$^{3}$

context->execute_task(launcher); 


\section{Processing Physical Regions}

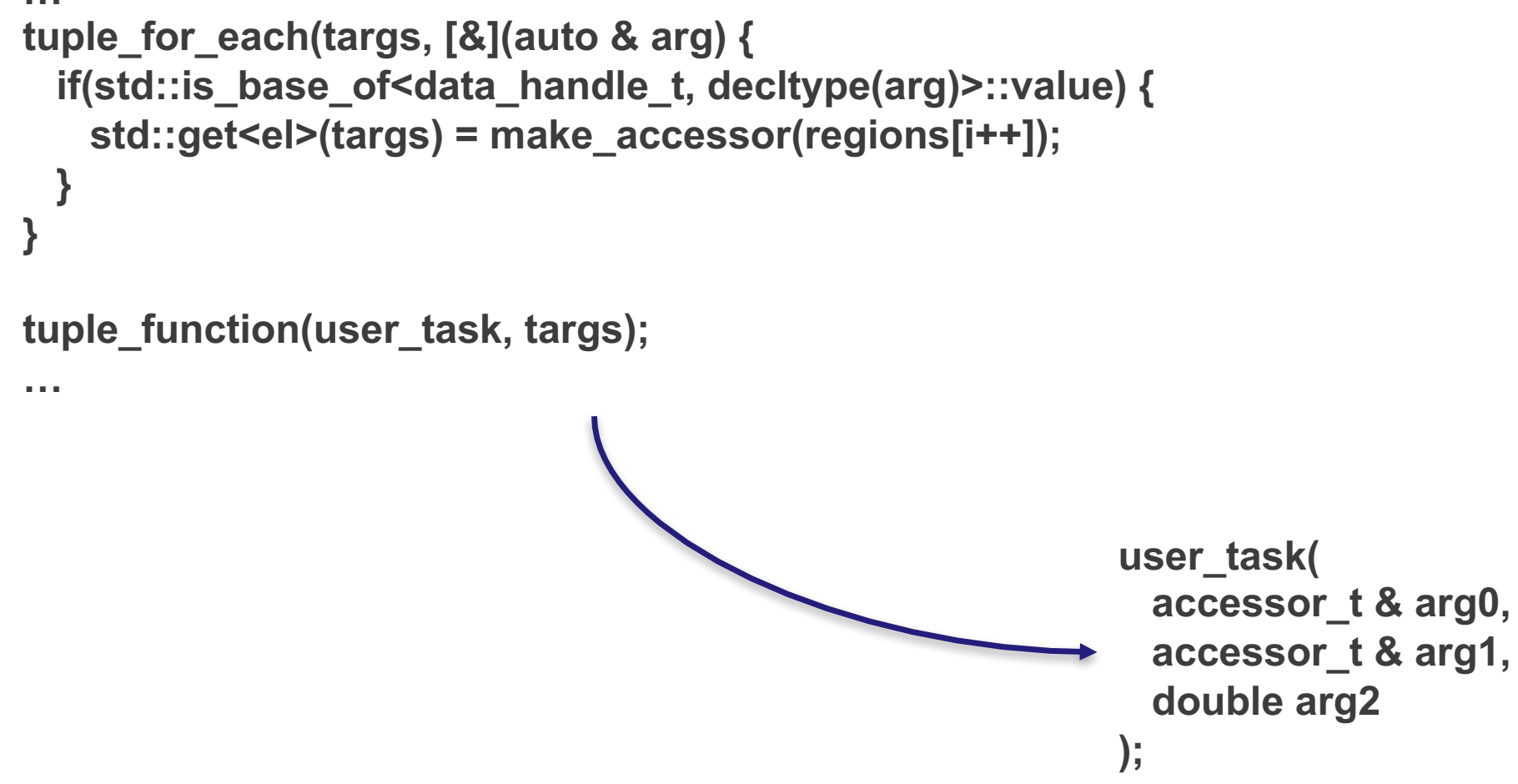

tuple_function(user_task, targs); 


\section{Legion Execution Workflow}




\section{Legion/MPI Initialization in FleCSI}

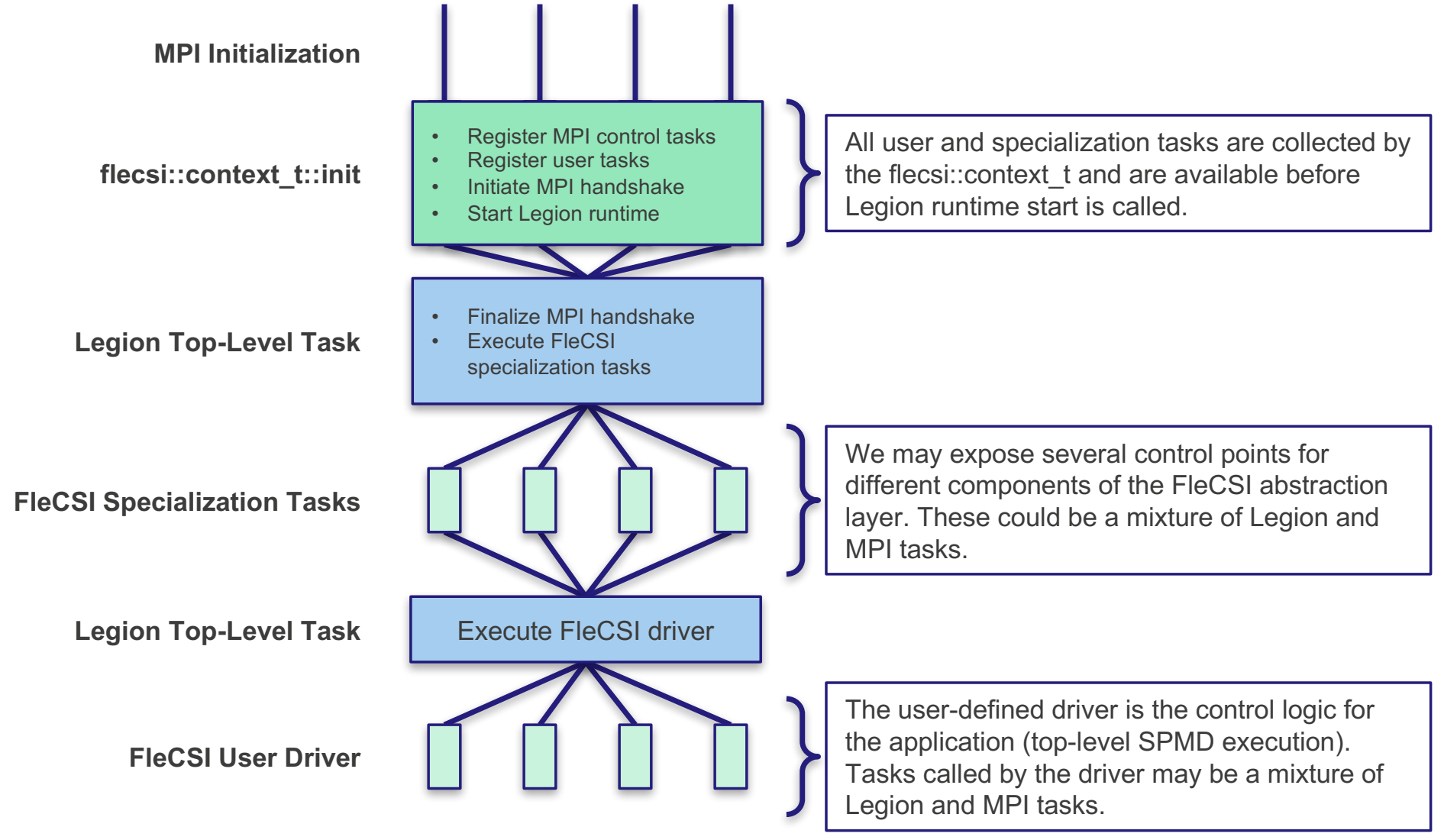




\section{FleCSI Mesh Description}




\section{What is a FleCSI Mesh?}

A FleCSI mesh_topology_t is a data structure that allows the storage and initialization of topological connectivities.

- A developer uses FleCSI to create mesh interfaces for application developers

- The mesh topology can be statically specialized to use different entity types and to store different connectivity information

- Clarification (definition): mesh domains separate mesh topologies that would otherwise have incompatible types, e.g., edges and corners have the same topological dimension and cannot reside in the same topology. 


\section{FleCSI meshes are composed of mesh} entities...

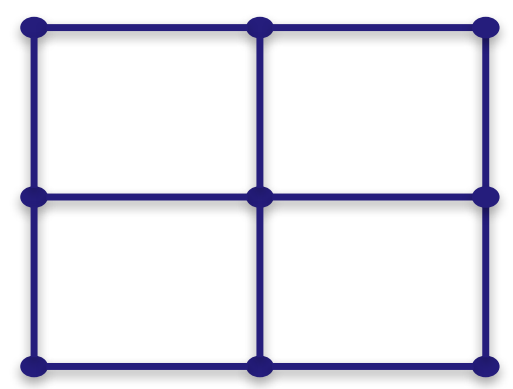




\section{FleCSI meshes are composed of mesh} entities...

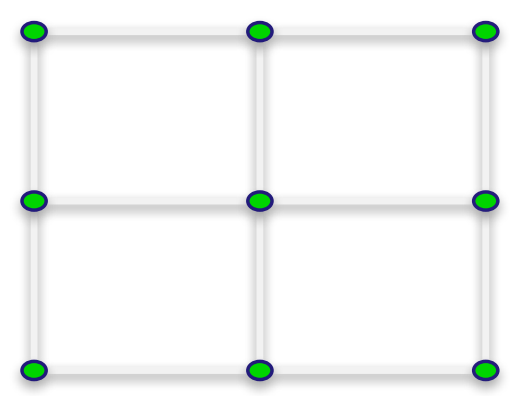

Vertices 


\title{
FleCSI meshes are composed of mesh
} entities...

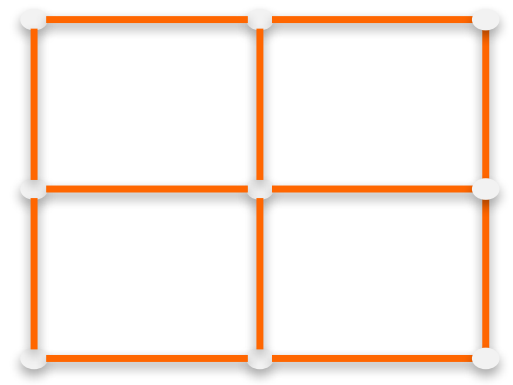

\author{
Edges
}




\title{
FleCSI meshes are composed of mesh
} entities...

\author{
Cells
}




\title{
FleCSI meshes are composed of mesh
} entities...

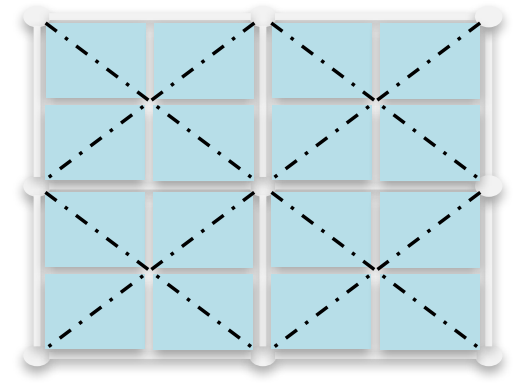

\author{
Corners
}




\section{Example mesh specialization types...}

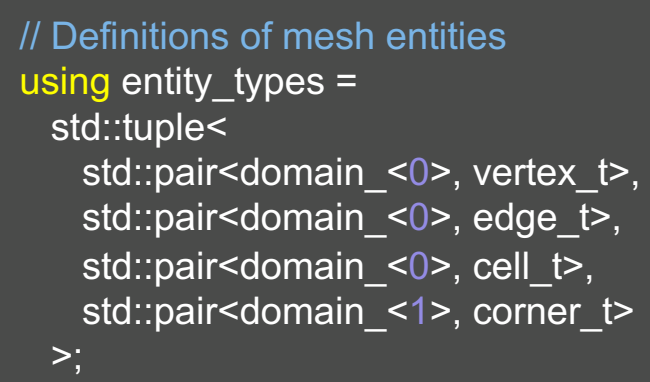

// Connectivities are adjacencies of entities within a single domain

using connectivities $=$

std::tuple<

std::tuple<domain_<0>, vertex_t, edge_t $t>$,

std::tuple<domain_<0>, vertex_t, cell_t $>$,

std::tuple $<$ domain_<0>, cell_t, vertex_t $>$

$>$;

// Bindings are adjacencies of entities across two domains

using bindings $=$

std::tuple<

std: :tuple<domain_<0>, domain_<1>, cell_t $\mathrm{t}$ corner_t $>$, std: :tuple $<$ domain_ $<0>$, domain_<1>, vertex_t, corner_t $>$ $>$; 


\section{FleCSI mesh entities can be enumerated to create Index Spaces}

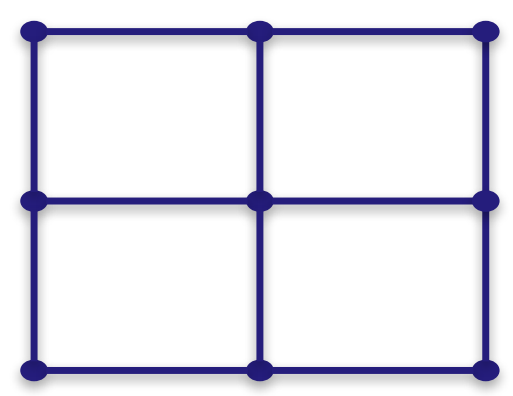




\section{FleCSI mesh entities can be enumerated to create Index Spaces}

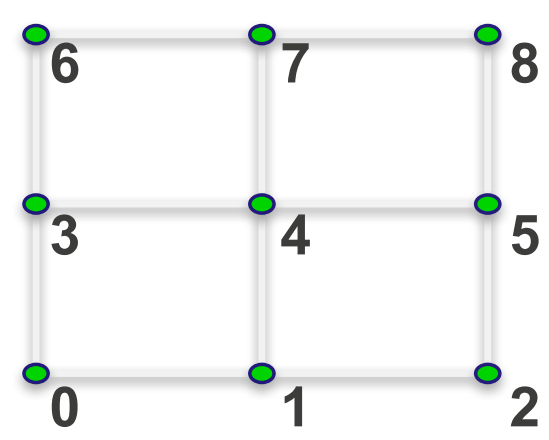

\section{Vertices}

$$
\{0,1,2,3,4,5,6,7,8\}
$$




\title{
FleCSI mesh entities can be enumerated to create Index Spaces
}

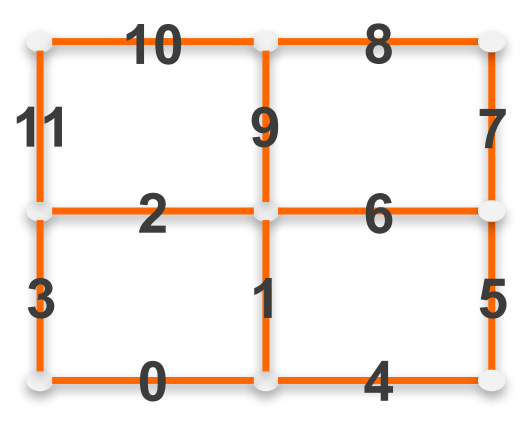

\author{
Edges \\ $\{0,1,2,3,4,5,6,7,8,9,10,11\}$
}




\title{
FleCSI mesh entities can be enumerated to create Index Spaces
}

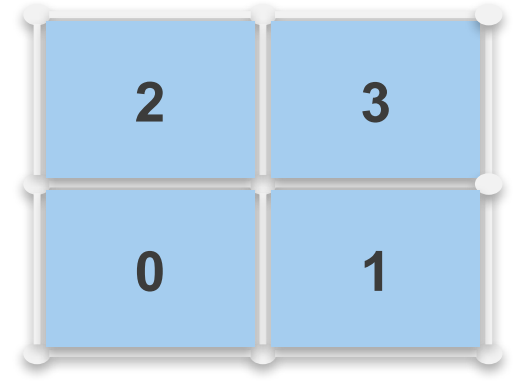

\author{
Cells \\ $\{0,1,2,3\}$
}




\section{FleCSI mesh entities can be enumerated to create Index Spaces}

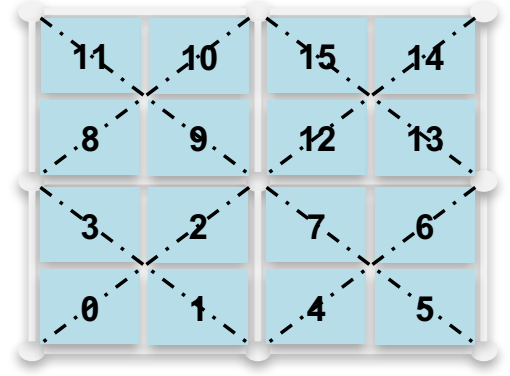

\section{Corners}

$$
\{0,1,2,3,4,5,6,7,8,9,10,11,12,13,14,15\}
$$




\section{The FleCSI interface separates access from data layout...}

- The entities of the mesh define iterable collections:

$$
\text { foreach (auto c: mesh.cells ()) ... }
$$

- The index spaces of the mesh define data attachment sites:

$$
\text { register_data(m, hydro, pressure, double, dense, 1, cells); }
$$

- Index spaces and data model map well to modern programming efforts like Legion 


\section{High-level Example}

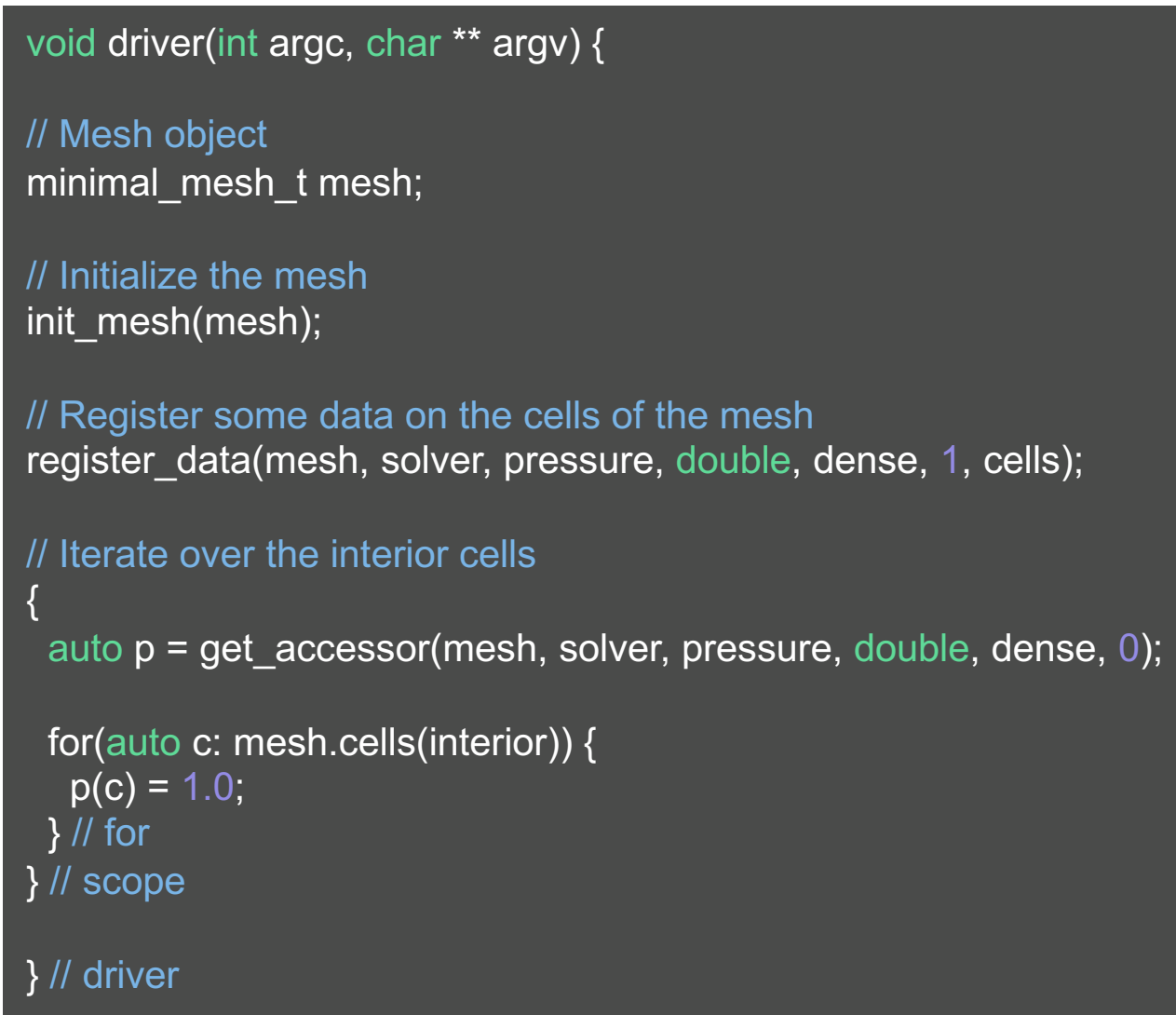




\section{The minimal_mesh_t uses FleCSI to implement the mesh interface...}

class minimal_mesh_t

: public topology::mesh_topology_t $<$ minimal_types_t $>\{$ public:

// Initialize the mesh topology. This is called after the specialization

// adds the mesh entities.

void init() \{

// Initialize domain 0 of the mesh topology.

topology::mesh_topology_t $<$ minimal_types_t $>::$ init $<0>()$;

// Other initialization things here, e.g. create special static index spaces...

\}// init

\}; // minimal_mesh_t 


\section{The minimal_mesh_t uses FleCSI to implement the mesh interface...}

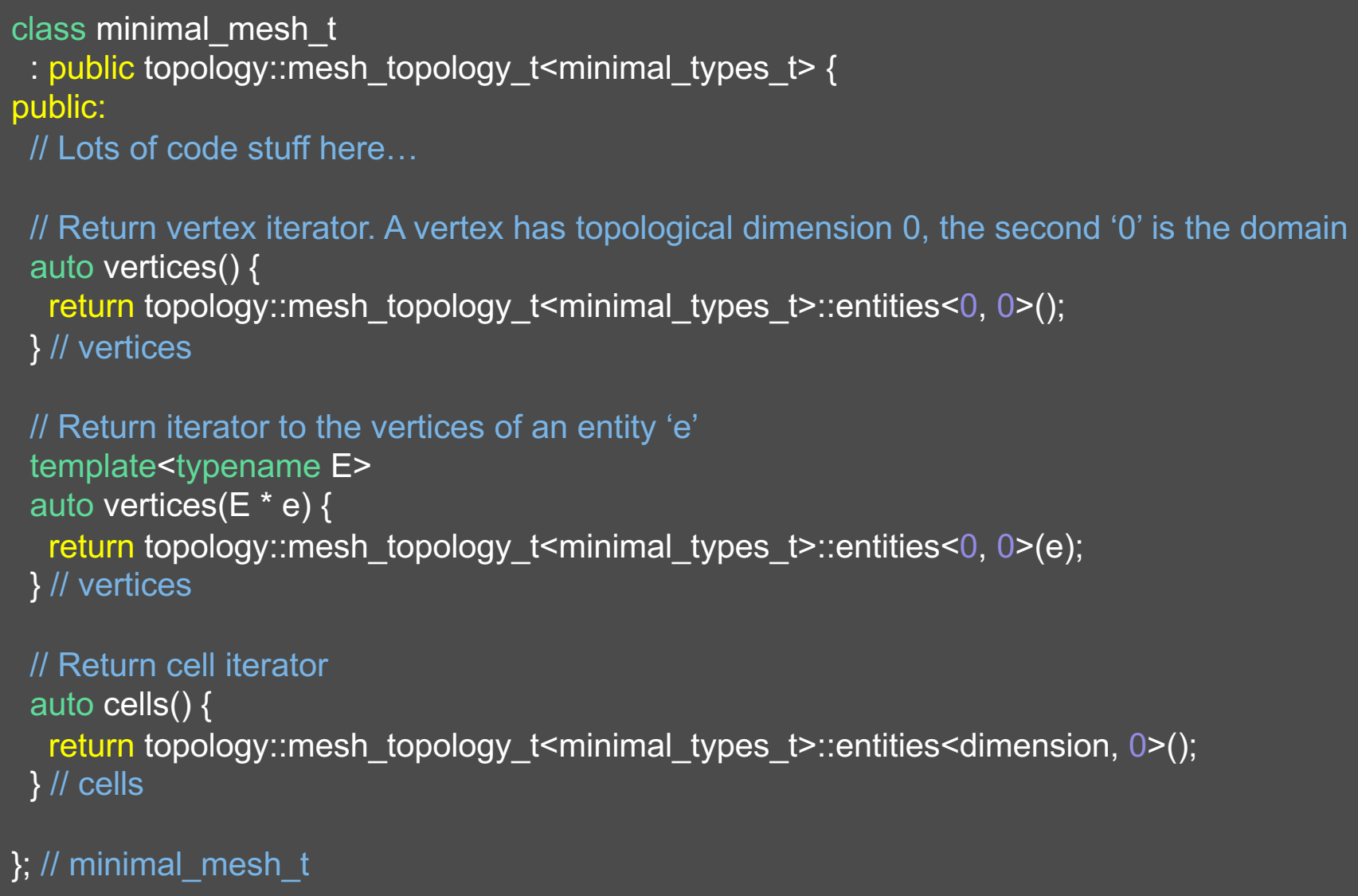




\section{The minimal_mesh_t uses FleCSI to implement the mesh interface...}

\section{class minimal_mesh_t}

: public topology::mesh_topology_t<minimal_types_t $>\{$ public:

// Lots of code stuff here...

// Return an iterator to a static subset of the cells (created in init())

auto cells(size_t is) \{

switch(is) \{

case minimal::interior:

return interior_cells_;

case minimal::boundary:

return boundary_cells_;

default:

assert(false \&\& "unknown index space");

\}// switch

\} // cells

\}; // minimal_mesh_t 


\section{The minimal_types_t type provides static specialization information to mesh_topology_t}

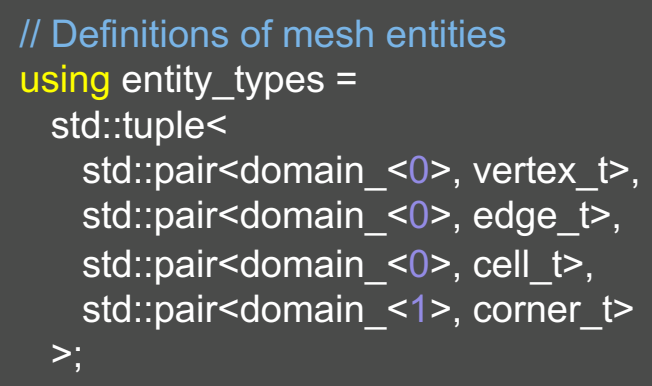

// Connectivities are adjacencies of entities within a single domain

using connectivities $=$

std::tuple<

std::tuple<domain_<0>, vertex_t, edge_t $t>$,

std::tuple<domain_<0>, vertex_t, cell_t $>$,

std::tuple<domain_<0>, cell_t, vertex_t $>$

$>$;

// Bindings are adjacencies of entities across two domains

using bindings $=$

std::tuple<

std::tuple<domain_<0>, domain_<1>, cell_t corner_t $>$,

std: :tuple $<$ domain_ $<0>$, domain_<1>, vertex_t, corner_t $>$ $>$; 


\section{Mesh Partitioning}




\section{How does FleCSI handle distributed memory?}

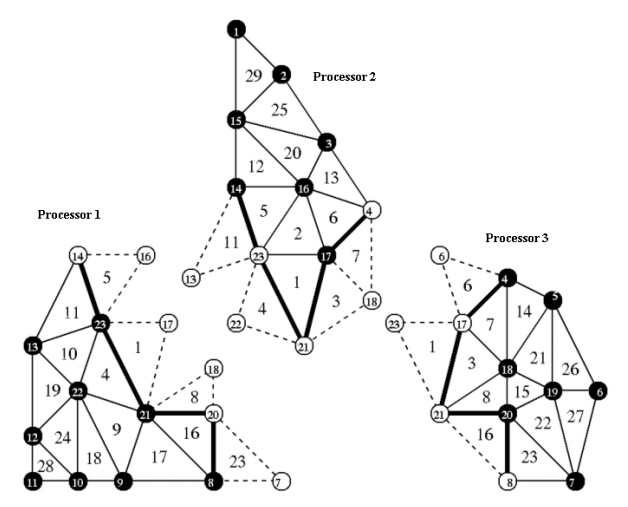

Mesh 


\section{How does FleCSI handle distributed memory?

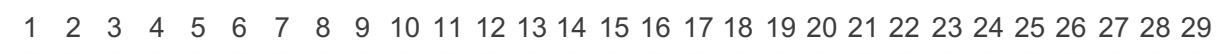 00000000000000000000000000000}

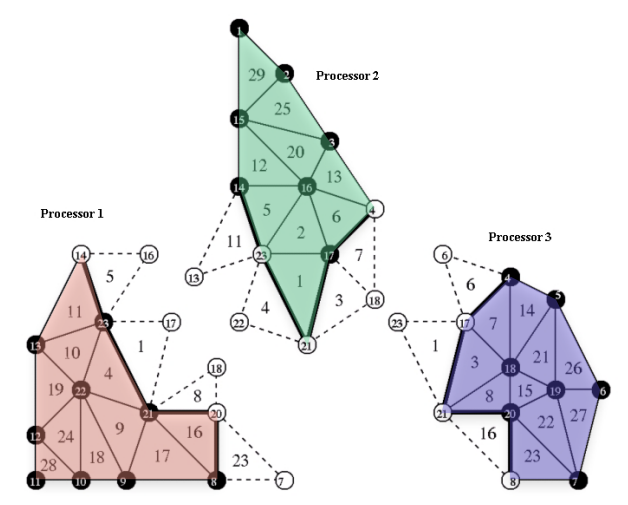

$\begin{array}{cccccccc} & 0 & 000 & 0000 & 0 & 0 & \text { primary } 1 \\ 0 & 00 & 0 & 0 & 0 & 0 & 0 & \text { primary } 2 \\ 0 & 00 & 000 & 0 & 0 & \text { primary } 3\end{array}$

\section{Mesh}

The mesh is partitioned by entities in one of the topological dimensions, e.g., cells. 


\section{How does
memory?}
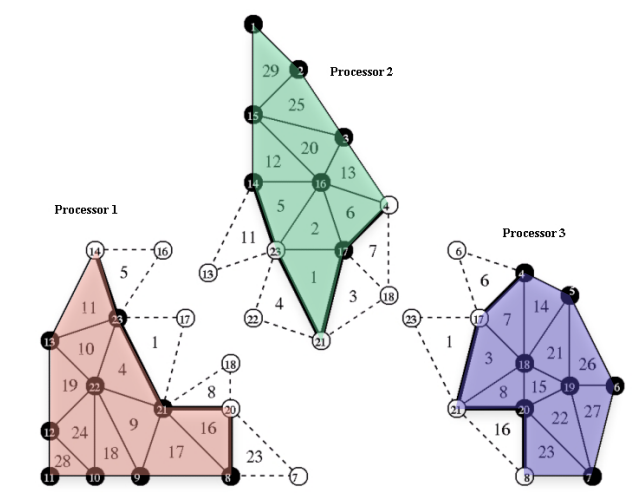

\section{Mesh}

The mesh is partitioned by entities in one of the topological dimensions, e.g., cells.
$1 \quad 2 \quad 3 \quad 4 \quad 5 \quad 6 \quad 7 \quad 8 \quad 9 \quad 1011121314151617181920212223242526272829$ 00000000000000000000000000000

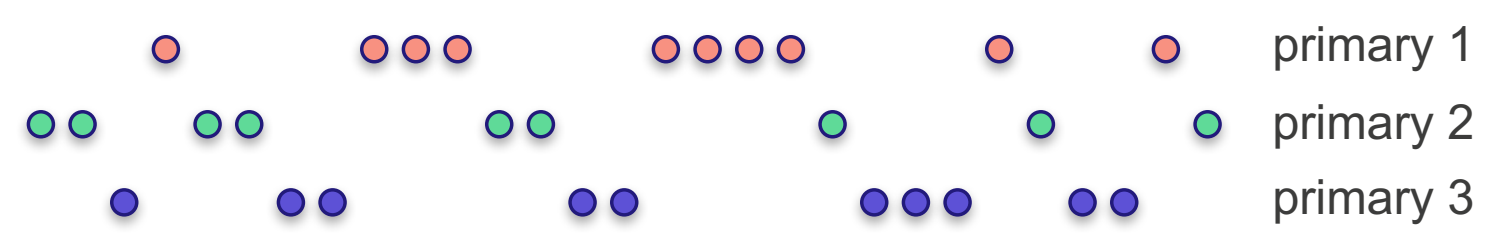

The primary partitioning splits the topology into contiguous sets of cells. 


\section{How does FleCSI handle distributed memory?

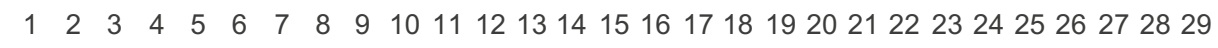 00000000000000000000000000000}

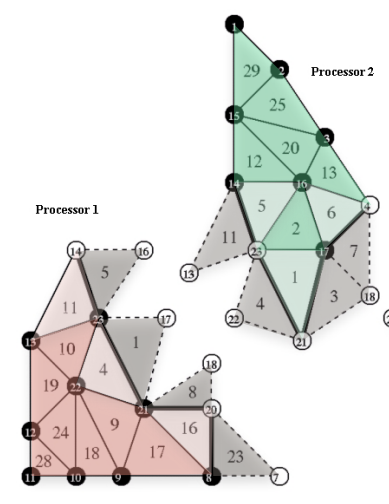

Mesh

Using a strategy defined by the specialization, the dependency closure of the mesh is formed, creating several sets of indices (index spaces).

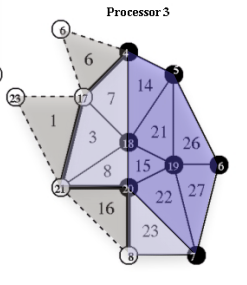

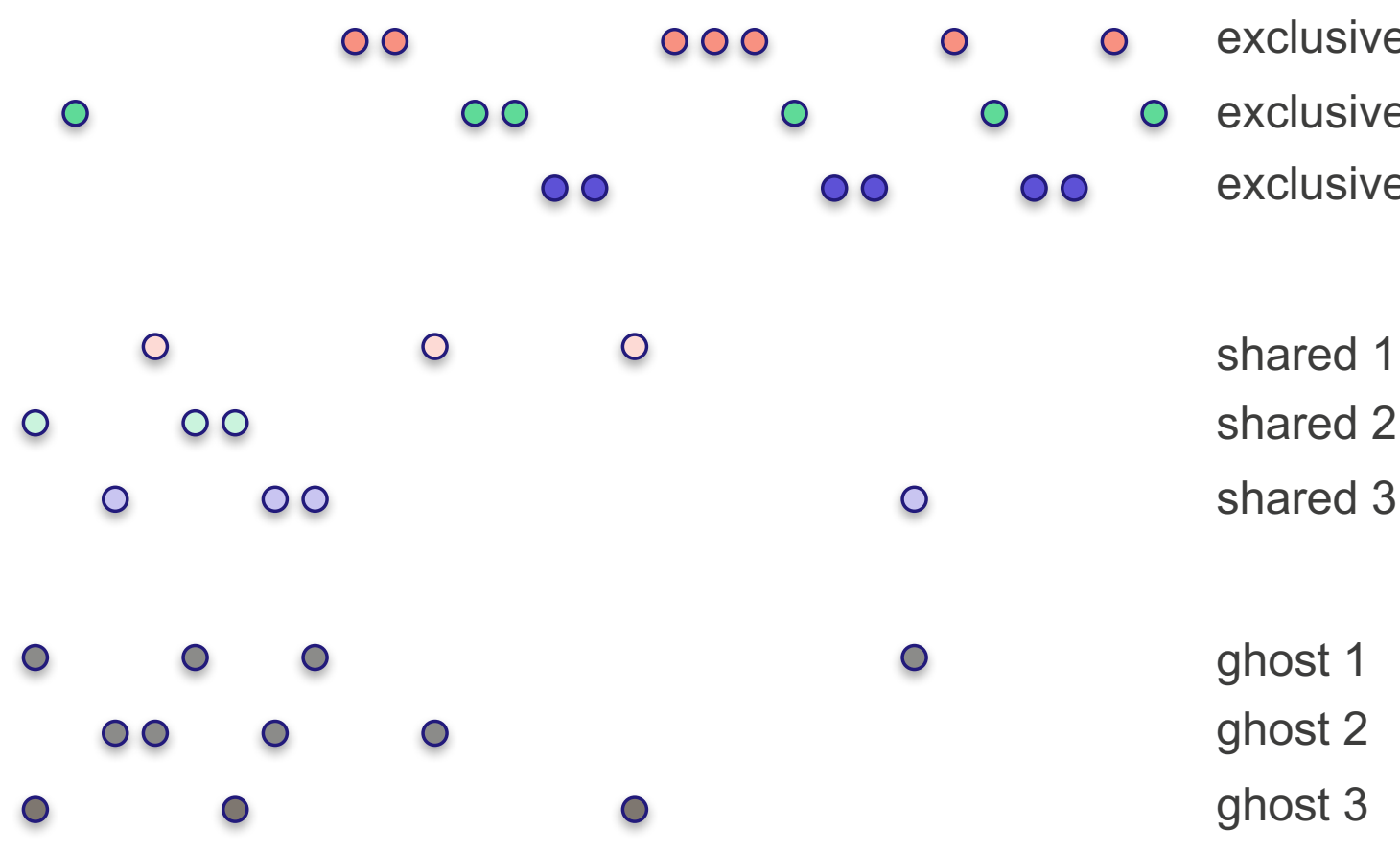

○

$\circ \quad 00$

$\begin{array}{cccc}0 & 0 & 0 & \\ 0 & 0 & 0 & 0\end{array}$ 


\section{How does
memory?}

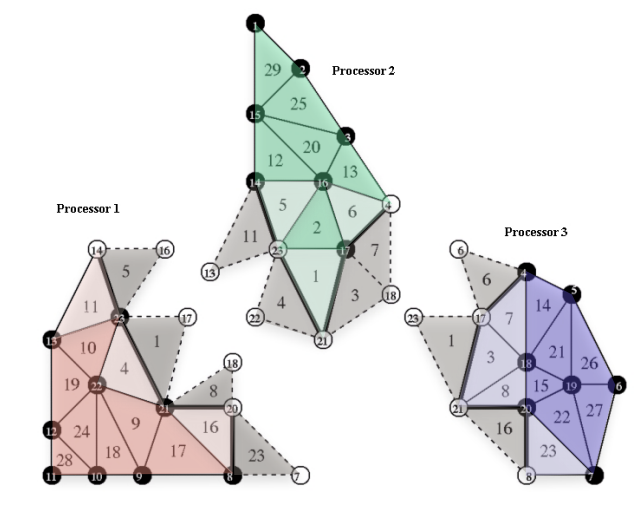

\section{Mesh}

Using a strategy defined by the specialization, the dependency closure of the mesh is formed, creating several sets of indices (index spaces).
$1 \quad 2 \quad 3 \quad 4 \quad 5 \quad 6 \quad 7 \quad 8 \quad 91011121314151617181920212223242526272829$ 00000000000000000000000000000
Exclusive: I own them, and nobody else cares about them

Shared: I own them, and some other people care about them

Ghost: I don't own them, but I care about them exclusive 1

exclusive 2

exclusive 3

shared 1

shared 2

shared 3

ghost 1

ghost 2

ghost 3 


\section{How does FleCSI handle distributed memory?

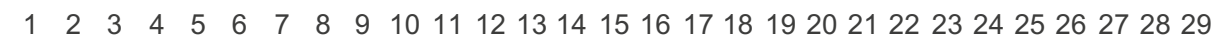 00000000000000000000000000000}

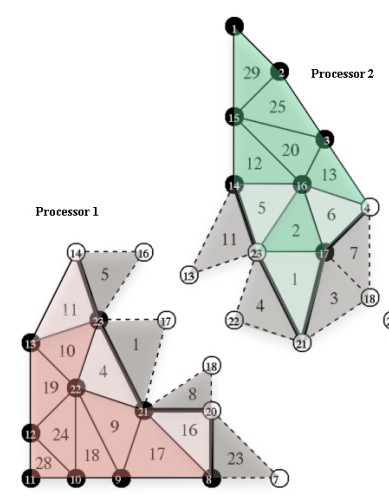

Mesh

Multiple partitionings and partition closure strategies can be employed within the same specialization.
○

0

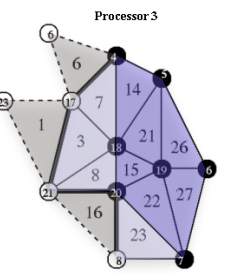

0

000
000

00

00

0

o

0

00

0

o

○ o

o 0

exclusive 3

shared 1

shared 2

shared 3

ghost 1

ghost 2

ghost 3 


\section{How does FleCSI handle distributed} memory?

$\begin{array}{lllllllllllll}1 & 2 & 3 & 4 & 5 & 6 & 7 & 8 & 9 & 1011 & 121314151617181920212223242526272829 \\ 0\end{array}$

00

000

O

○

exclusive 1

O

0

o

shared 1

\section{Mesh}

On each SPMD task (rank), the

O

00

○

ghost 1 closure forms a set of virtual index spaces that represent a complete set of dependency data. 


\section{How does FleCSI handle distributed} memory?

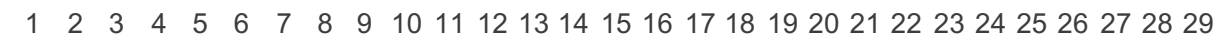 00000000000000000000000000000

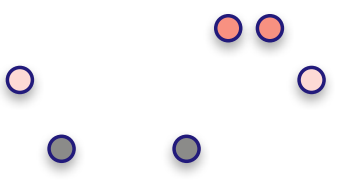
000
○
○
0
O
- exclusive 1 shared 1 ghost 1

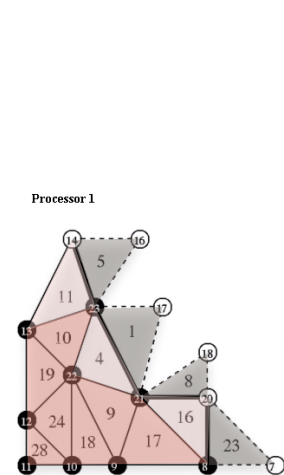

\section{Mesh}

The virtual index spaces can be iterated using foreach semantics. 


\section{How does FleCSI handle distributed} memory?

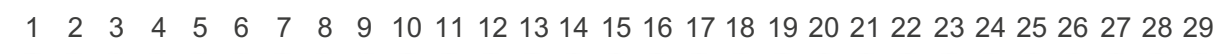
00000000000000000000000000000

\begin{tabular}{|c|c|c|c|c|c|}
\hline \multirow[b]{3}{*}{0} & 00 & \multirow{2}{*}{$0^{000}$} & \multirow[t]{2}{*}{0} & \multirow[t]{3}{*}{0} & \multirow{3}{*}{$\begin{array}{l}\text { exclusive } 1 \\
\text { shared } 1 \\
\text { ghost } 1\end{array}$} \\
\hline & 0 & & & & \\
\hline & 0 & & 0 & & \\
\hline 00 & 0000 & 0000 & 00 & 0 & \\
\hline
\end{tabular}

foreach(auto c: mesh.cells()) \{// traverse all cells (union of sets $O$ )

\section{Mesh}

The virtual index spaces can be iterated using foreach semantics. 


\section{How does FleCSI handle distributed} memory?

\section{$1 \quad 2 \quad 3 \quad 4 \quad 5 \quad 6 \quad 7 \quad 8 \quad 91011121314151617181920212223242526272829$ 00000000000000000000000000000}

\begin{tabular}{|c|c|c|c|c|c|c|}
\hline \multirow[b]{3}{*}{0} & & 00 & 000 & 0 & 0 & exclusive 1 \\
\hline & 0 & 0 & 0 & & & shared 1 \\
\hline & 0 & 0 & & & & ghost 1 \\
\hline & 0 & 000 & 0000 & 0 & 0 & \\
\hline
\end{tabular}

foreach(auto c: mesh.cells(local)) \{ // traverse cells in the // union of exclusive and // shared 0

\section{Mesh}

The virtual index spaces can be iterated using foreach semantics. 


\section{How does FleCSI handle distributed} memory?

$\begin{array}{lllllllllllll}1 & 2 & 3 & 4 & 5 & 6 & 7 & 8 & 9 & 1011 & 121314151617181920212223242526272829 \\ 0\end{array}$

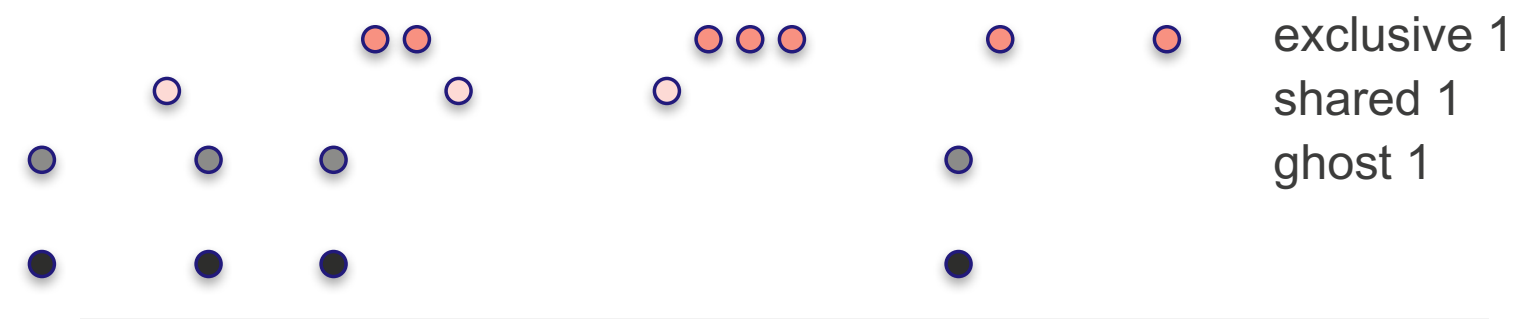

foreach(auto c: mesh.cells(ghost)) \{// traverse cells in the ghost set $\bullet$

\section{Mesh}

The virtual index spaces can be iterated using foreach semantics. 


\section{How does FleCSI handle distributed} memory?

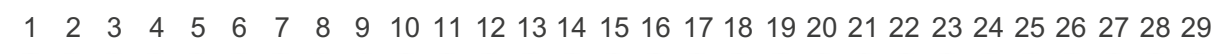
00000000000000000000000000000

\begin{tabular}{|c|c|c|c|c|c|}
\hline & 00 & 000 & 0 & 0 & exclusive 1 \\
\hline 0 & 0 & 0 & & & shared 1 \\
\hline 0 & 0 & & 0 & & ghost 1 \\
\hline
\end{tabular}

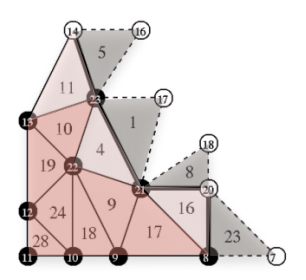

○

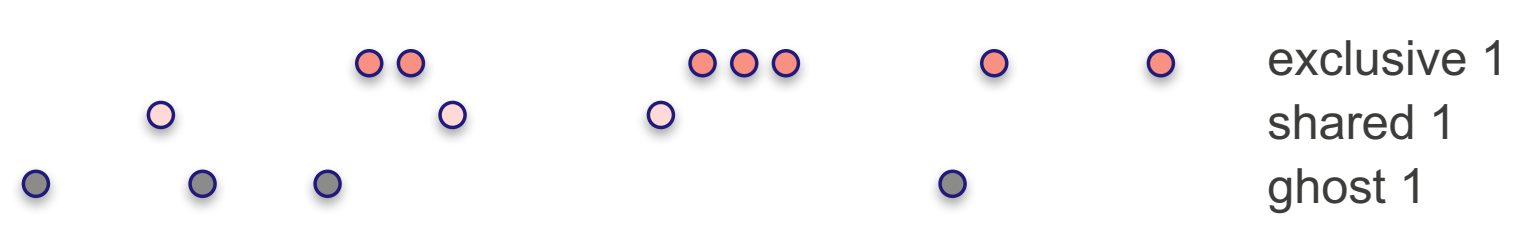

\section{Mesh}

This provides a clean interface for complex data access and execution that handles dependency updates using permissions specified for the

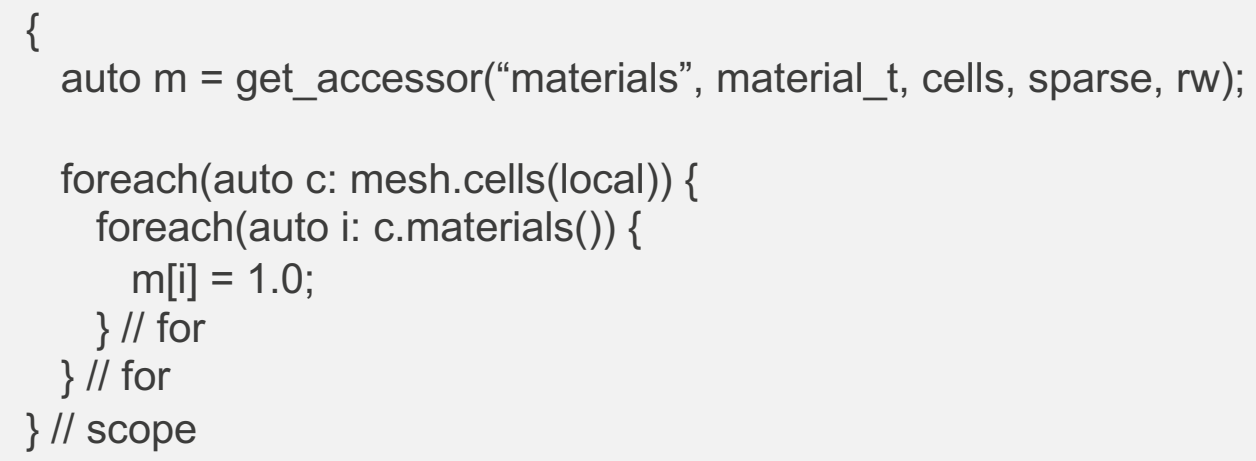




\section{How does FleCSI use Legion for dependency updates?}
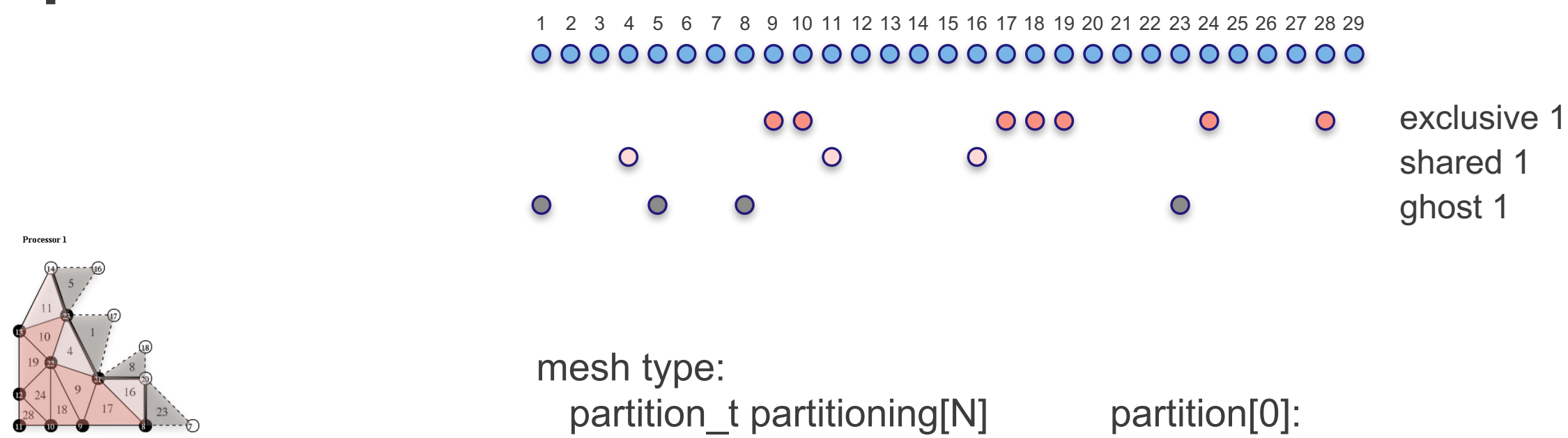

mesh type:

partition_t partitioning[N]

partition[0]:

IndexSpace exclusive 1

Mesh

The local mesh stores the partition information as several index spaces using an IndexPartition

(a Legion C++ type). 


\section{How do the topology and data models work together for Legion?}

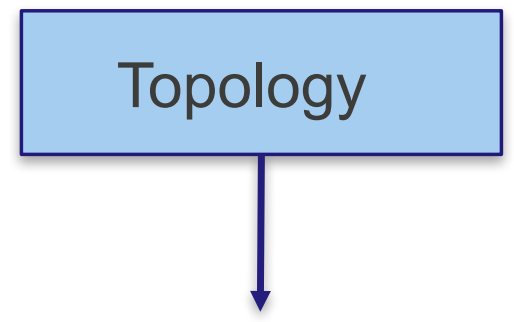

Index

Space(s)

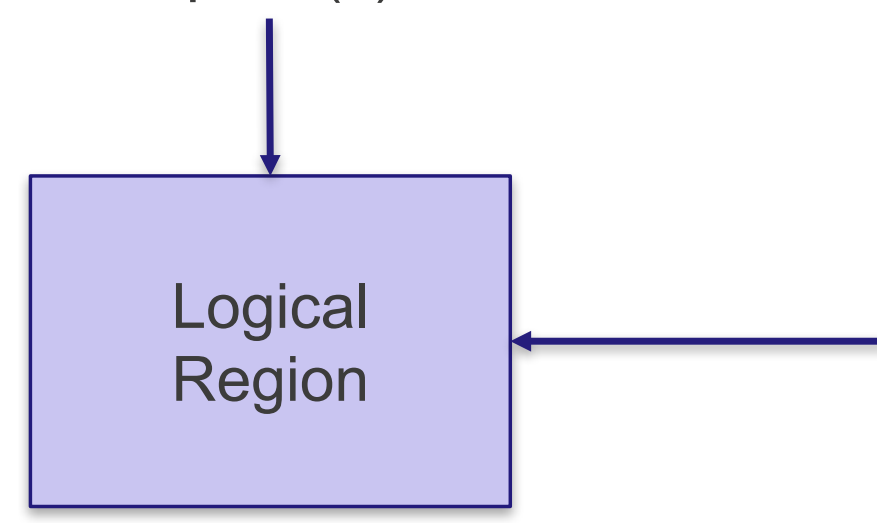

Field

Space(s)
Data Manager 


\section{How do the topology and data models work together for Legion?}

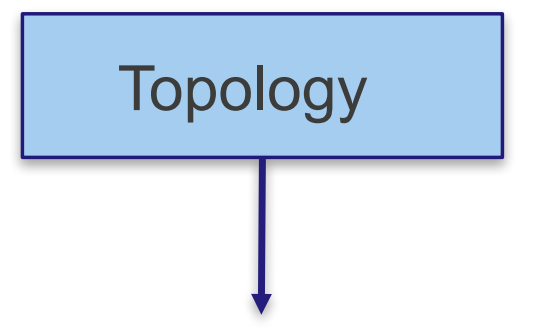

Index

Space(s)

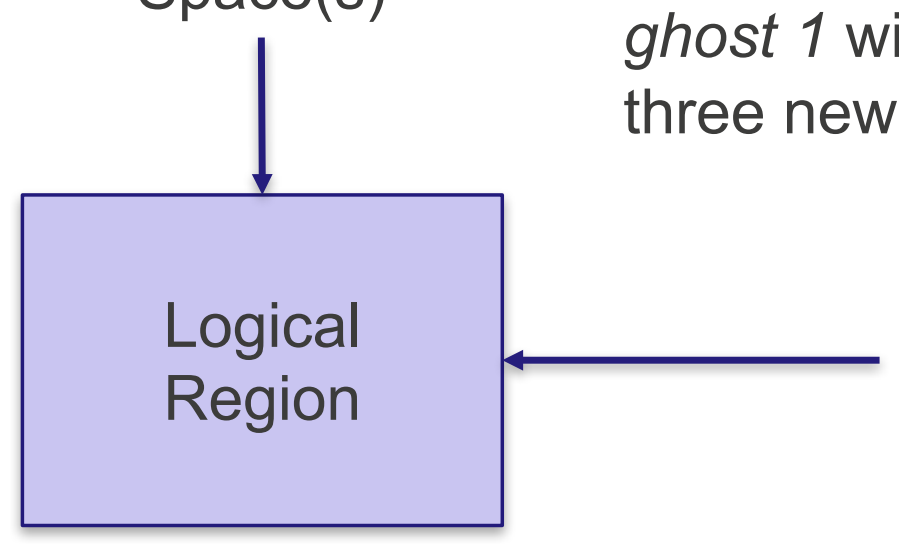

\{

\}// scope
When a user registers data, the data manager and topology cooperate to create several logical regions...

register_data("pressure", double, cells, dense);

The Legion backend will use exclusive 1, shared 1, and ghost 1 with new field spaces of type double to create three new logical regions.

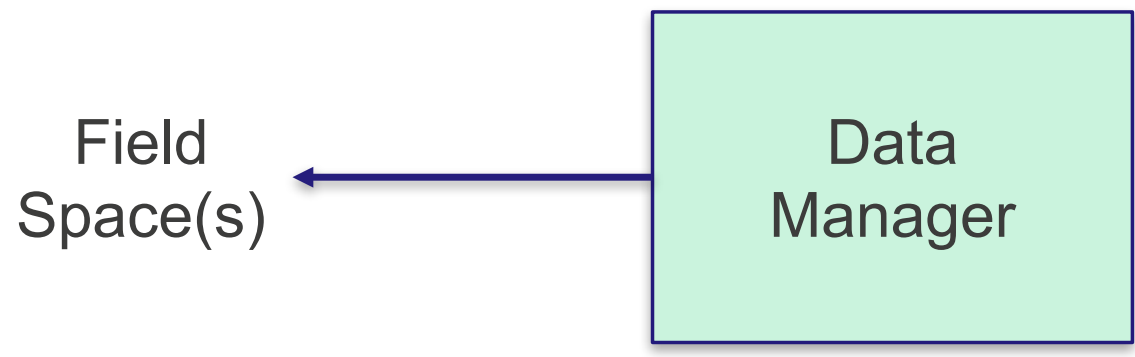




\section{How do the topology and data models work together for Legion?}

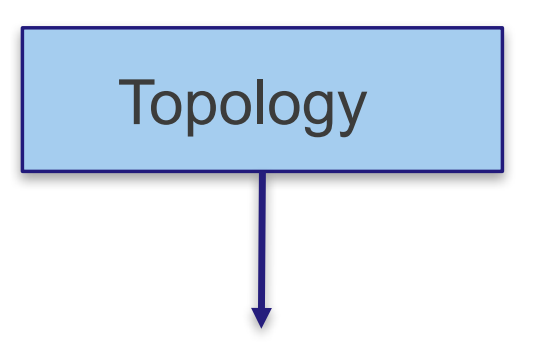

Index Space(s)

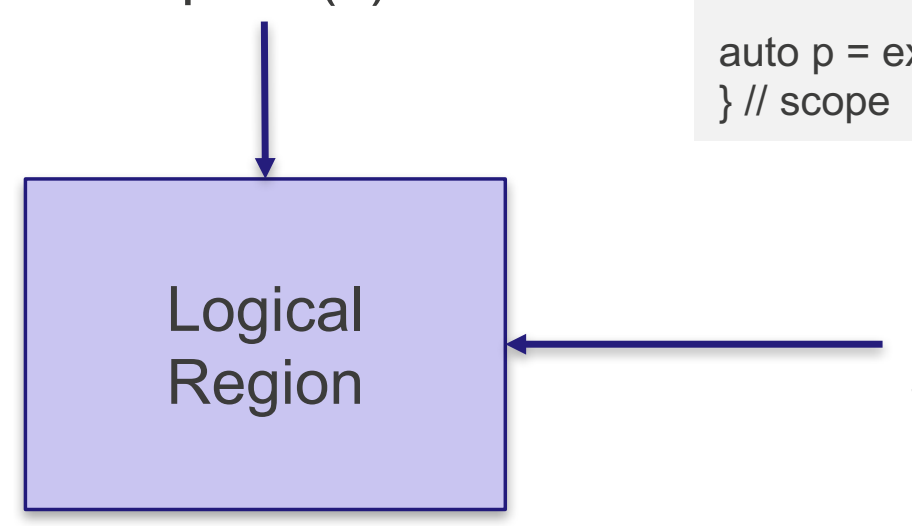

\{

\}// scope
FleCSI tasks operate transparently on Legion logical regions using compile-time data handles that are available through the FleCSI interface: auto $r$ = get_handle("density", double, cells, local, dense, ro); auto e = get_handle("internal energy", double, cells, local, dense, ro); auto $p=$ get_handle("pressure", double, cells, local, dense, rw);

auto $p=$ execute_task $(e o s, r, e)$;

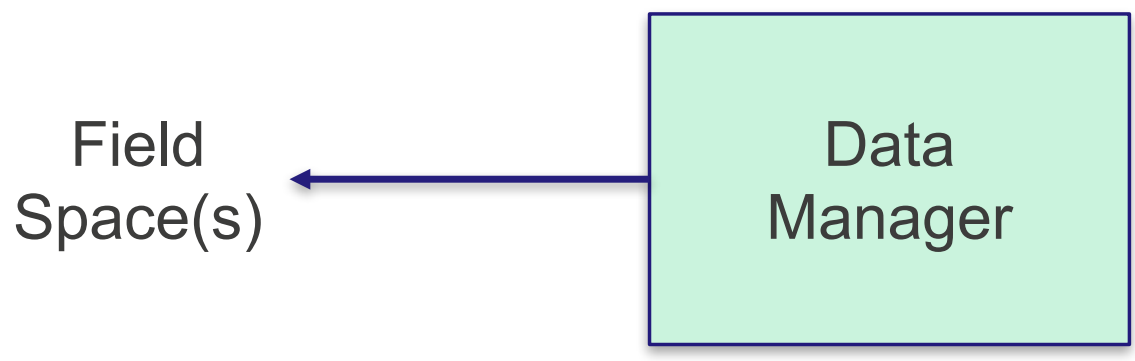




\section{Legion Partitioning (old interface)}


Cells Index Space $\quad 122345567891011121314151617181920212223242526272829$

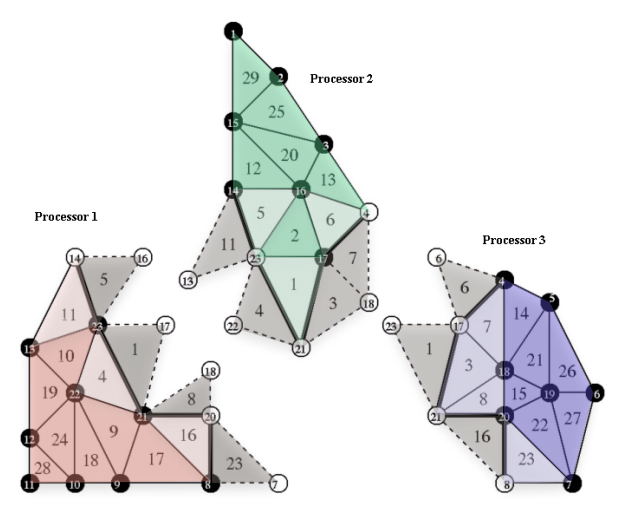

Mesh
00000000000000000000000000000

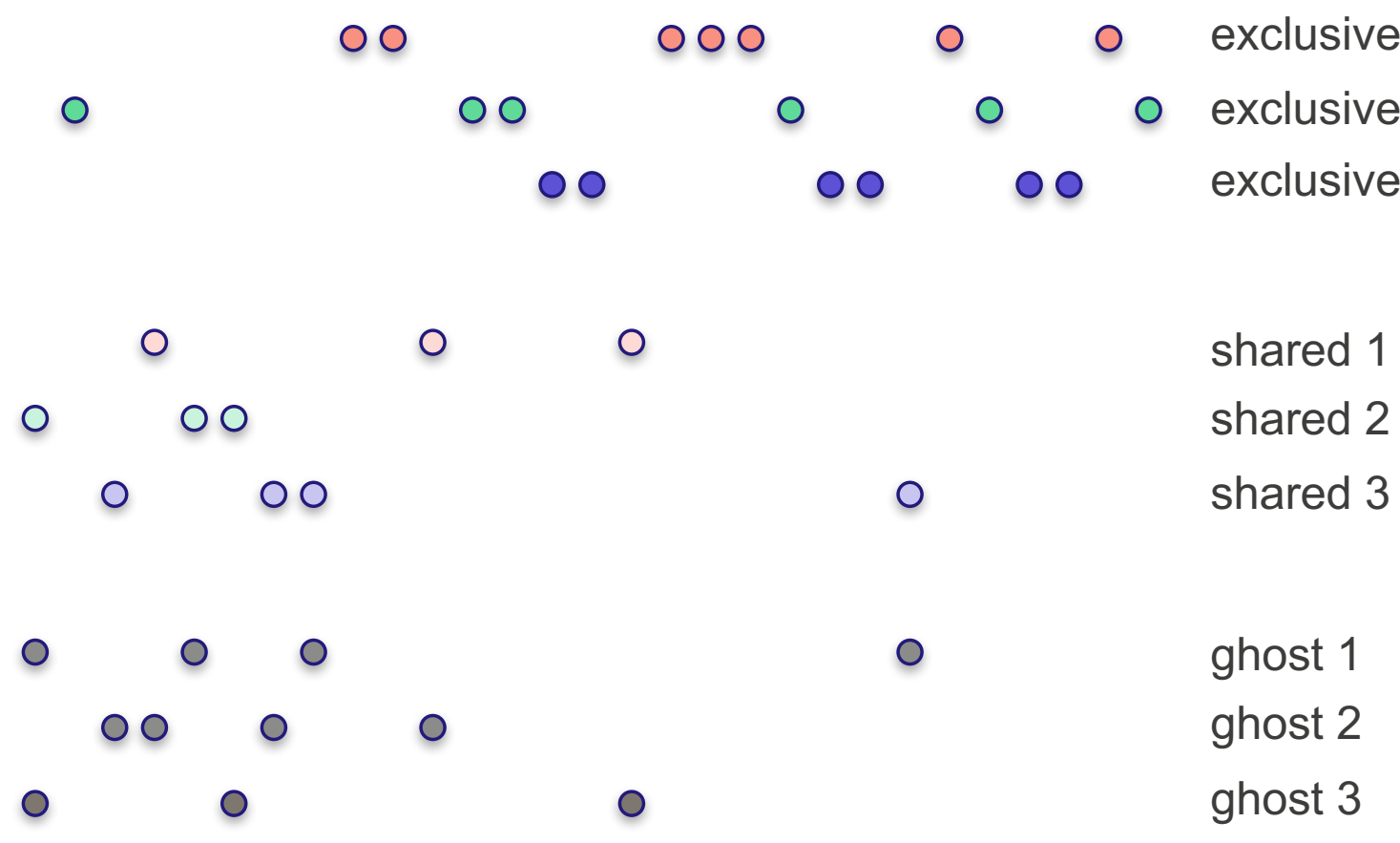




\section{Mesh Cells IS $\rightarrow$ Global Cells IS}

$\left.\begin{array}{l}\{4,9,10,11,16,17,18,19,24,28,1,2,5,6,12,13,20,25,29,3,7,8,14,15,21,22,23,26,27\} \\ \{0,1,2,3,4,5,6,7,8,9,10,11,12,13,14,15,16,17,18,19,20,21,22,23,24,25,26,27,28\end{array}\right\}$ 


\section{Mesh Cells IS $\rightarrow$ Global Cells IS}

primary 1: $\{4,9,10,11,16,17,18,19,24,28\}$ actual 1: $\{0,1,2,3,4,5,6,7,8,9\}$

primary $2:\{1,2,5,6,12,13,20,25,29\}$ actual 2: $\{10,11,12,13,14,15,16,17,18\}$

primary 3: $\{3,7,8,14,15,21,22,23,26,27\}$ actual 3: $\{19,20,21,22,23,24,25,26,27,28\}$

ghost 1: $\{1,5,8,23\}$ actual 1: $\{10,12,21,26\}$

ghost 2: $\{3,4,7,11\}$ actual $2:\{19,0,20,3\}$

ghost 3: $\{1,6,16\}$ actual 3: $\{10,13,4\}$

\section{Index Partitions (Global IS indices)}

exclusive $1:\{1,2,5,6,7,8,9\}$

shared 1: $\{0,3,4\}$

ghost 1: $\{10,12,21,26\}$

exclusive 2 : $\{11,14,15,16,17,18\}$ shared 2: $\{10,12,13\}$ ghost 2: $\{19,0,20,3\}$

exclusive 3: $\{22,23,24,25,27,28\}$ shared 3: $\{19,20,21,26\}$ ghost 3: $\{10,13,4\}$ 
Vertices Index Space $\quad \begin{aligned} & 1234567891011121314151617181920212223 \\ & 00000000000000000000000\end{aligned}$

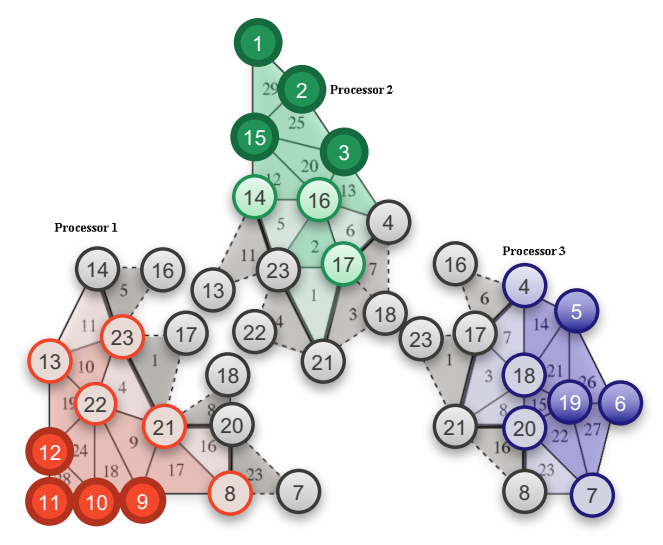

Mesh

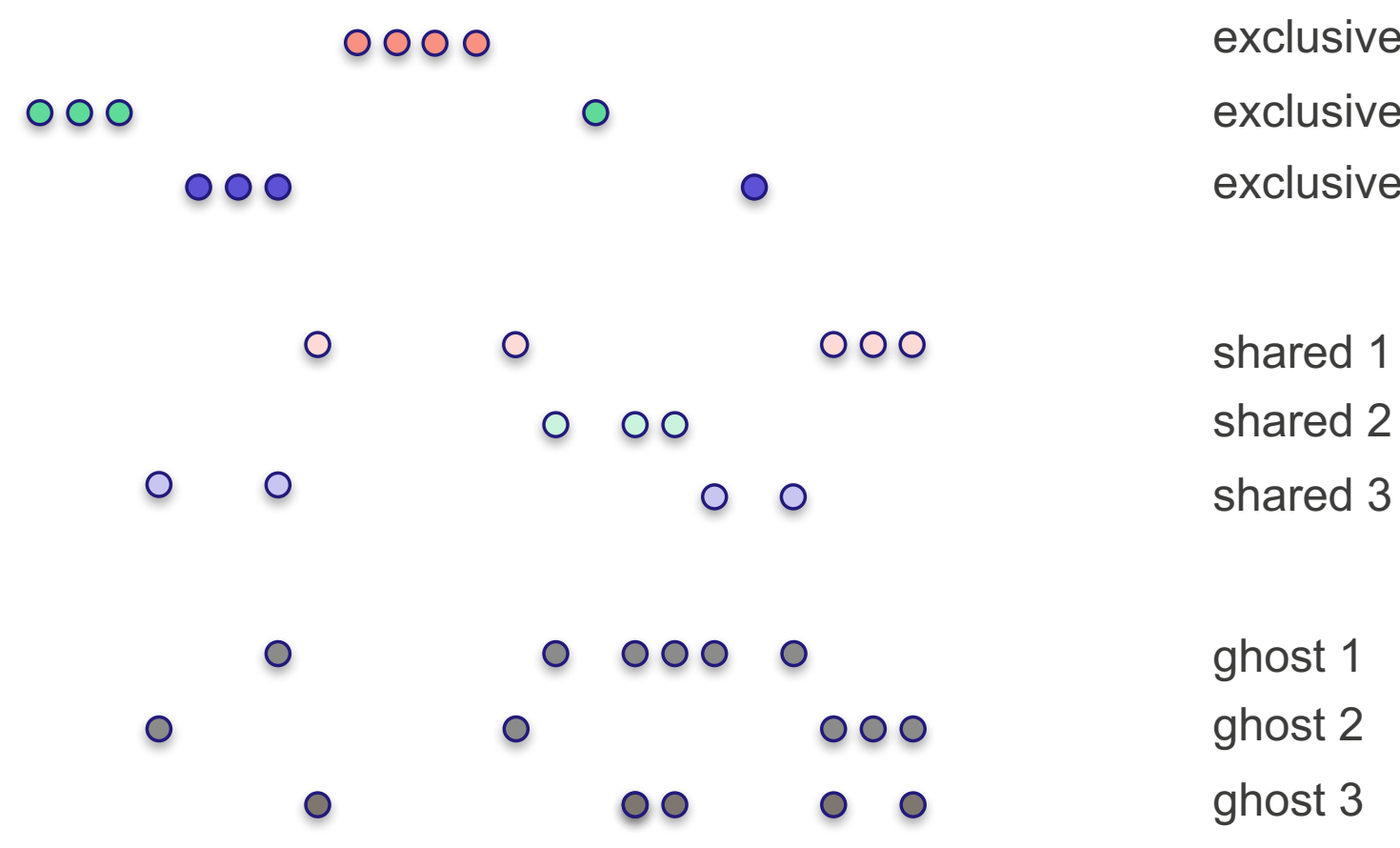




\section{Mesh Vertices IS $\rightarrow$ Global Vertices IS}

$\{8,9,10,11,12,13,21,22,23,1,2,3,14,15,16,17,4,5,6,7,18,19,20\}$
$\{0,1,2,3,4,5,6,7,8,9,10,11,12,13,14,15,16,17,18,19,20,21,22\}$ 


\section{Mesh Vertices IS $\rightarrow$ Global Vertices IS}

primary 1: $\{8,9,10,11,12,13,21,22,23\}$ actual 1: $\{0,1,2,3,4,5,6,7,8\}$

primary $2:\{1,2,3,14,15,16,17\}$ actual 2: $\{9,10,11,12,13,14,15\}$

primary $3:\{4,5,6,7,18,19,20\}$ actual 3: $\{16,17,18,19,20,21,22\}$

ghost 1: $\{14,16,17,18,7\}$ actual $1:\{12,14,15,20,19\}$

ghost 2: $\{13,22,23,21,18,4\}$ actual $2:\{5,7,8,6,20,16\}$

ghost 3: $\{16,17,23,21,8\}$ actual 3: $\{14,15,8,6,0\}$

\section{Index Partitions (Global IS indices)}

exclusive $1:\{1,2,3,4\}$ shared 1: $\{0,5,6,7,8\}$ ghost 1: $\{12,14,15,19,20\}$

exclusive 2: $\{9,10,11,13\}$ shared 2: $\{12,14,15\}$ ghost 2: $\{5,6,7,8,16,20\}$

exclusive $3:\{17,18,19\}$ shared 3: $\{16,19,20,22\}$ ghost 3: $\{0,6,8,14,15\}$ 


\section{Representing connectivity in a dependent partitioning friendly way}

- These are homogenous polygons so we could simply have a field for each vertex, but imagine if these polygons are heterogeneous or we are representing vertex to edge connectivity where a single vertex could be incident on many edges.

\section{Mesh Cells IS $\rightarrow$ Global Cells IS}

$\left\{\begin{array}{l}4,9,10,11,16,17,18,19,24, \ldots . \\ 0,1,2,3,4,5,6,7,8, \ldots\}\end{array}\right\}$

We add a field over the Global Cells IS which references ranges in another index space "D"

$\{\quad 0: 2, \quad 3: 5, \quad 6: 8, \quad 9: 11,12: 14,15: 17,18: 20,21: 23,24: 26 \ldots \ldots .$.$\} \quad IS C x Field<Range> Vertices$

We create an index space D and add a field over this IS that points to elements in the Global Vertices IS

$\left\{\begin{array}{l}6,7,8,1,7,6, \quad 5,7,8,5,8,12,0,6,22, \quad 0,1,6, \quad 1,2,7, \quad 4,5,7, \quad 2,4,7, \ldots \ldots \ldots . .\end{array} \quad\right.$ IS D x Field<ptr_t $>$ vertices

\section{Mesh Vertices IS $\rightarrow$ Global Vertices IS}

$\{8,9,10,11,12,13,21,22,23,1,2,3,14,15,16,17,4,5,6,7,18,19,20\}$

$\{0,1,2,3,4,5,6,7,8,9,10,11,12,13,14,15,16,17,18,19,20,21,22\}$ 


\section{Simple $8 \times 8$ mesh}

\begin{tabular}{|c|c|c|c|c|c|c|c|}
\hline 56 & 57 & 58 & 59 & 60 & 61 & 62 & 63 \\
\hline 48 & 49 & 50 & 51 & 52 & 53 & 54 & 55 \\
\hline 40 & 41 & 42 & 43 & 44 & 45 & 46 & 47 \\
\hline 32 & 33 & 34 & 35 & 36 & 37 & 38 & 39 \\
\hline 24 & 25 & 26 & 27 & 28 & 29 & 30 & 31 \\
\hline 16 & 17 & 18 & 19 & 20 & 21 & 22 & 23 \\
\hline 8 & 9 & 10 & 11 & 12 & 13 & 14 & 15 \\
\hline 0 & 1 & 2 & 3 & 4 & 5 & 6 & 7 \\
\hline
\end{tabular}




\section{Creating the primary partition...}

// Create a graph definition from file. The simple_definition_t type maps the // information in the file, but does not read the mesh definition into memory. flecsi::topology::simple_definition_t gd("simple2d-8x8.msh");

// Create the dCRS representation for the distributed partitioner. auto dcrs = flecsi::dmp::make_dcrs(gd);

// Create a partitioner instance to generate the primary partition. auto partitioner $=$ std:::make_shared $<$ flecsi:::dmp:::parmetis_partitioner_t $>()$;

// Create the primary partition.

auto primary = partitioner->partition(dcrs); 


\section{Cell 0 Adjacencies}

\section{dCRS for rank 0}

offsets: 0258111417202225293337 indices: 180291310241135124613

57146150916181017

2911183101219

distribution: 01225385164

\begin{tabular}{|c|c|c|c|c|c|c|c|}
\hline 56 & 57 & 58 & 59 & 60 & 61 & 62 & 63 \\
\hline 48 & 49 & 50 & 51 & 52 & 53 & 54 & 55 \\
\hline 40 & 41 & 42 & 43 & 44 & 45 & 46 & 47 \\
\hline 32 & 33 & 34 & 35 & 36 & 37 & 38 & 39 \\
\hline 24 & 25 & 26 & 27 & 28 & 29 & 30 & 31 \\
\hline 16 & 17 & 18 & 19 & 20 & 21 & 22 & 23 \\
\hline 8 & 9 & 10 & 11 & 12 & 13 & 14 & 15 \\
\hline 0 & 1 & 2 & 3 & 4 & 5 & 6 & 7 \\
\hline
\end{tabular}




\section{Cell 1 Adjacencies}

\section{dCRS for rank 0}

offsets: 0258111417202225293337 indices: 180291310241135124613

57146150916181017

2911183101219

distribution: 01225385164

\begin{tabular}{|c|c|c|c|c|c|c|c|}
\hline 56 & 57 & 58 & 59 & 60 & 61 & 62 & 63 \\
\hline 48 & 49 & 50 & 51 & 52 & 53 & 54 & 55 \\
\hline 40 & 41 & 42 & 43 & 44 & 45 & 46 & 47 \\
\hline 32 & 33 & 34 & 35 & 36 & 37 & 38 & 39 \\
\hline 24 & 25 & 26 & 27 & 28 & 29 & 30 & 31 \\
\hline 16 & 17 & 18 & 19 & 20 & 21 & 22 & 23 \\
\hline 8 & 9 & 10 & 11 & 12 & 13 & 14 & 15 \\
\hline 0 & 1 & 2 & 3 & 4 & 5 & 6 & 7 \\
\hline
\end{tabular}




\section{Cell 2 Adjacencies}

\section{dCRS for rank 0}

offsets: 0258111417202225293337 indices: 180291310241135124613

57146150916181017

2911183101219

distribution: 01225385164

\begin{tabular}{|c|c|c|c|c|c|c|c|}
\hline 56 & 57 & 58 & 59 & 60 & 61 & 62 & 63 \\
\hline 48 & 49 & 50 & 51 & 52 & 53 & 54 & 55 \\
\hline 40 & 41 & 42 & 43 & 44 & 45 & 46 & 47 \\
\hline 32 & 33 & 34 & 35 & 36 & 37 & 38 & 39 \\
\hline 24 & 25 & 26 & 27 & 28 & 29 & 30 & 31 \\
\hline 16 & 17 & 18 & 19 & 20 & 21 & 22 & 23 \\
\hline 8 & 9 & 10 & 11 & 12 & 13 & 14 & 15 \\
\hline 0 & 1 & 2 & 3 & 4 & 5 & 6 & 7 \\
\hline
\end{tabular}




\section{Cell 3 Adjacencies}

\section{dCRS for rank 0}

offsets: 0258111417202225293337 indices: 180291310241135124613

57146150916181017

2911183101219

distribution: 01225385164

\begin{tabular}{|c|c|c|c|c|c|c|c|}
\hline 56 & 57 & 58 & 59 & 60 & 61 & 62 & 63 \\
\hline 48 & 49 & 50 & 51 & 52 & 53 & 54 & 55 \\
\hline 40 & 41 & 42 & 43 & 44 & 45 & 46 & 47 \\
\hline 32 & 33 & 34 & 35 & 36 & 37 & 38 & 39 \\
\hline 24 & 25 & 26 & 27 & 28 & 29 & 30 & 31 \\
\hline 16 & 17 & 18 & 19 & 20 & 21 & 22 & 23 \\
\hline 8 & 9 & 10 & 11 & 12 & 13 & 14 & 15 \\
\hline 0 & 1 & 2 & 3 & 4 & 5 & 6 & 7 \\
\hline
\end{tabular}




\section{Cell 4 Adjacencies}

\section{dCRS for rank 0}

offsets: 0258111417202225293337 indices: 180291310241135124613

57146150916181017

2911183101219

distribution: 01225385164

\begin{tabular}{|c|c|c|c|c|c|c|c|}
\hline 56 & 57 & 58 & 59 & 60 & 61 & 62 & 63 \\
\hline 48 & 49 & 50 & 51 & 52 & 53 & 54 & 55 \\
\hline 40 & 41 & 42 & 43 & 44 & 45 & 46 & 47 \\
\hline 32 & 33 & 34 & 35 & 36 & 37 & 38 & 39 \\
\hline 24 & 25 & 26 & 27 & 28 & 29 & 30 & 31 \\
\hline 16 & 17 & 18 & 19 & 20 & 21 & 22 & 23 \\
\hline 8 & 9 & 10 & 11 & 12 & 13 & 14 & 15 \\
\hline 0 & 1 & 2 & 3 & 4 & 5 & 6 & 7 \\
\hline
\end{tabular}




\section{Cell 5 Adjacencies}

\section{dCRS for rank 0}

offsets: 0258111417202225293337 indices: 180291310241135124613

57146150916181017

2911183101219

distribution: 01225385164

\begin{tabular}{|c|c|c|c|c|c|c|c|}
\hline 56 & 57 & 58 & 59 & 60 & 61 & 62 & 63 \\
\hline 48 & 49 & 50 & 51 & 52 & 53 & 54 & 55 \\
\hline 40 & 41 & 42 & 43 & 44 & 45 & 46 & 47 \\
\hline 32 & 33 & 34 & 35 & 36 & 37 & 38 & 39 \\
\hline 24 & 25 & 26 & 27 & 28 & 29 & 30 & 31 \\
\hline 16 & 17 & 18 & 19 & 20 & 21 & 22 & 23 \\
\hline 8 & 9 & 10 & 11 & 12 & 13 & 14 & 15 \\
\hline 0 & 1 & 2 & 3 & 4 & 5 & 6 & 7 \\
\hline
\end{tabular}




\section{Cell 6 Adjacencies}

\section{dCRS for rank 0}

offsets: 0258111417202225293337 indices: 180291310241135124613

57146150916181017

2911183101219

distribution: 01225385164

\begin{tabular}{|c|c|c|c|c|c|c|c|}
\hline 56 & 57 & 58 & 59 & 60 & 61 & 62 & 63 \\
\hline 48 & 49 & 50 & 51 & 52 & 53 & 54 & 55 \\
\hline 40 & 41 & 42 & 43 & 44 & 45 & 46 & 47 \\
\hline 32 & 33 & 34 & 35 & 36 & 37 & 38 & 39 \\
\hline 24 & 25 & 26 & 27 & 28 & 29 & 30 & 31 \\
\hline 16 & 17 & 18 & 19 & 20 & 21 & 22 & 23 \\
\hline 8 & 9 & 10 & 11 & 12 & 13 & 14 & 15 \\
\hline 0 & 1 & 2 & 3 & 4 & 5 & 6 & 7 \\
\hline
\end{tabular}




\section{Cell 7 Adjacencies}

\section{dCRS for rank 0}

offsets: 0258111417202225293337 indices: 180291310241135124613

57146150916181017

2911183101219

distribution: 01225385164

\begin{tabular}{|c|c|c|c|c|c|c|c|}
\hline 56 & 57 & 58 & 59 & 60 & 61 & 62 & 63 \\
\hline 48 & 49 & 50 & 51 & 52 & 53 & 54 & 55 \\
\hline 40 & 41 & 42 & 43 & 44 & 45 & 46 & 47 \\
\hline 32 & 33 & 34 & 35 & 36 & 37 & 38 & 39 \\
\hline 24 & 25 & 26 & 27 & 28 & 29 & 30 & 31 \\
\hline 16 & 17 & 18 & 19 & 20 & 21 & 22 & 23 \\
\hline 8 & 9 & 10 & 11 & 12 & 13 & 14 & 15 \\
\hline 0 & 1 & 2 & 3 & 4 & 5 & 6 & 7 \\
\hline
\end{tabular}




\section{Cell 8 Adjacencies}

\section{dCRS for rank 0}

offsets: 0258111417202225293337 indices: 180291310241135124613

57146150916181017

2911183101219

distribution: 01225385164

\begin{tabular}{|c|c|c|c|c|c|c|c|}
\hline 56 & 57 & 58 & 59 & 60 & 61 & 62 & 63 \\
\hline 48 & 49 & 50 & 51 & 52 & 53 & 54 & 55 \\
\hline 40 & 41 & 42 & 43 & 44 & 45 & 46 & 47 \\
\hline 32 & 33 & 34 & 35 & 36 & 37 & 38 & 39 \\
\hline 24 & 25 & 26 & 27 & 28 & 29 & 30 & 31 \\
\hline 16 & 17 & 18 & 19 & 20 & 21 & 22 & 23 \\
\hline 8 & 9 & 10 & 11 & 12 & 13 & 14 & 15 \\
\hline 0 & 1 & 2 & 3 & 4 & 5 & 6 & 7 \\
\hline
\end{tabular}




\section{Cell 9 Adjacencies}

\section{dCRS for rank 0}

offsets: 0258111417202225293337 indices: 180291310241135124613

57146150916181017

2911183101219

distribution: 01225385164

\begin{tabular}{|c|c|c|c|c|c|c|c|}
\hline 56 & 57 & 58 & 59 & 60 & 61 & 62 & 63 \\
\hline 48 & 49 & 50 & 51 & 52 & 53 & 54 & 55 \\
\hline 40 & 41 & 42 & 43 & 44 & 45 & 46 & 47 \\
\hline 32 & 33 & 34 & 35 & 36 & 37 & 38 & 39 \\
\hline 24 & 25 & 26 & 27 & 28 & 29 & 30 & 31 \\
\hline 16 & 17 & 18 & 19 & 20 & 21 & 22 & 23 \\
\hline 8 & 9 & 10 & 11 & 12 & 13 & 14 & 15 \\
\hline 0 & 1 & 2 & 3 & 4 & 5 & 6 & 7 \\
\hline
\end{tabular}




\section{Cell 10 Adjacencies}

\section{dCRS for rank 0}

offsets: 0258111417202225293337 indices: 180291310241135124613

57146150916181017

2911183101219

distribution: 01225385164

\begin{tabular}{|c|c|c|c|c|c|c|c|}
\hline 56 & 57 & 58 & 59 & 60 & 61 & 62 & 63 \\
\hline 48 & 49 & 50 & 51 & 52 & 53 & 54 & 55 \\
\hline 40 & 41 & 42 & 43 & 44 & 45 & 46 & 47 \\
\hline 32 & 33 & 34 & 35 & 36 & 37 & 38 & 39 \\
\hline 24 & 25 & 26 & 27 & 28 & 29 & 30 & 31 \\
\hline 16 & 17 & 18 & 19 & 20 & 21 & 22 & 23 \\
\hline 8 & 9 & 10 & 11 & 12 & 13 & 14 & 15 \\
\hline 0 & 1 & 2 & 3 & 4 & 5 & 6 & 7 \\
\hline
\end{tabular}




\section{Cell 11 Adjacencies}

\section{dCRS for rank 0}

offsets: 0258111417202225293337 indices: 180291310241135124613

57146150916181017

2911183101219

distribution: 01225385164

\begin{tabular}{|c|c|c|c|c|c|c|c|}
\hline 56 & 57 & 58 & 59 & 60 & 61 & 62 & 63 \\
\hline 48 & 49 & 50 & 51 & 52 & 53 & 54 & 55 \\
\hline 40 & 41 & 42 & 43 & 44 & 45 & 46 & 47 \\
\hline 32 & 33 & 34 & 35 & 36 & 37 & 38 & 39 \\
\hline 24 & 25 & 26 & 27 & 28 & 29 & 30 & 31 \\
\hline 16 & 17 & 18 & 19 & 20 & 21 & 22 & 23 \\
\hline 8 & 9 & 10 & 11 & 12 & 13 & 14 & 15 \\
\hline 0 & 1 & 2 & 3 & 4 & 5 & 6 & 7 \\
\hline
\end{tabular}




\section{Creating the primary partition...}

// Create the dCRS representation for the distributed partitioner. auto dcrs = flecsi:::dmp:: make_dcrs $(g d)$;

// Create a partitioner instance to generate the primary partition. auto partitioner = std::make_shared<flecsi::dmp::parmetis_partitioner_t $>()$;

// Create the primary partition.

auto primary = partitioner->partition(dcrs); 


\section{What ParMETIS gives you back...}

$\square$ rank 0

$\square$ rank 1

$\square$ rank 2

$\square$ rank 3

$\square$ rank 4

\begin{tabular}{|c|c|c|c|c|c|c|c|}
\hline 56 & 57 & 58 & 59 & 60 & 61 & 62 & 63 \\
\hline 48 & 49 & 50 & 51 & 52 & 53 & 54 & 55 \\
\hline 40 & 41 & 42 & 43 & 44 & 45 & 46 & 47 \\
\hline 32 & 33 & 34 & 35 & 36 & 37 & 38 & 39 \\
\hline 24 & 25 & 26 & 27 & 28 & 29 & 30 & 31 \\
\hline 16 & 17 & 18 & 19 & 20 & 21 & 22 & 23 \\
\hline 8 & 9 & 10 & 11 & 12 & 13 & 14 & 15 \\
\hline 0 & 1 & 2 & 3 & 4 & 5 & 6 & 7 \\
\hline
\end{tabular}




\section{Simple $8 \times 8$ mesh partitioned with 5 ranks}

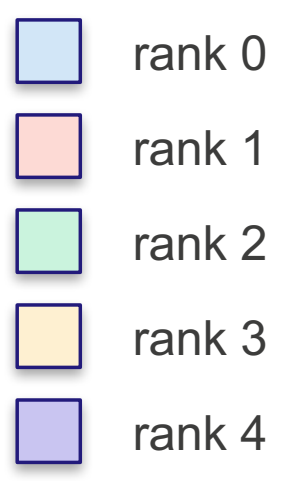

\begin{tabular}{|c|c|c|c|c|c|c|c|}
\hline 56 & 57 & 58 & 59 & 60 & 61 & 62 & 63 \\
\hline 48 & 49 & 50 & 51 & 52 & 53 & 54 & 55 \\
\hline 40 & 41 & 42 & 43 & 44 & 45 & 46 & 47 \\
\hline 32 & 33 & 34 & 35 & 36 & 37 & 38 & 39 \\
\hline 24 & 25 & 26 & 27 & 28 & 29 & 30 & 31 \\
\hline 16 & 17 & 18 & 19 & 20 & 21 & 22 & 23 \\
\hline 8 & 9 & 10 & 11 & 12 & 13 & 14 & 15 \\
\hline 0 & 1 & 2 & 3 & 4 & 5 & 6 & 7 \\
\hline
\end{tabular}




\section{Forming the dependency closure...}

// Compute the dependency closure of the primary cell partition

// through vertex intersections (specified by last template argument " 0 ").

// First two template arguments are "2" (cell) to "2" (cell) in 2D.

// To specify edge or face intersections, use 1 (edges) or 2 (faces in 3D).

auto closure = flecsi::topology::entity_closure<2,2,0>(gd, primary);

// Subtracting out the initial set leaves just the nearest

$/ /$ neighbors. This is similar to the image of the adjacency

// graph of the initial indices.

auto nearest_neighbors = flecsi::utils::set_difference(closure, primary); 


\section{Rank 0: primary + nearest neighbors}

$\square$ rank 0

rank 1

rank 2

rank 3

rank 4

\begin{tabular}{|c|c|c|c|c|c|c|c|}
\hline 56 & 57 & 58 & 59 & 60 & 61 & 62 & 63 \\
\hline 48 & 49 & 50 & 51 & 52 & 53 & 54 & 55 \\
\hline 40 & 41 & 42 & 43 & 44 & 45 & 46 & 47 \\
\hline 32 & 33 & 34 & 35 & 36 & 37 & 38 & 39 \\
\hline 24 & 25 & 26 & 27 & 28 & 29 & 30 & 31 \\
\hline 16 & 17 & 18 & 19 & 20 & 21 & 22 & 23 \\
\hline 8 & 9 & 10 & 11 & 12 & 13 & 14 & 15 \\
\hline 0 & 1 & 2 & 3 & 4 & 5 & 6 & 7 \\
\hline
\end{tabular}




\section{Rank 0: nearest neighbors}

$\square$ rank 0

rank 1

rank 2

rank 3

rank 4

\begin{tabular}{|c|c|c|c|c|c|c|c|}
\hline 56 & 57 & 58 & 59 & 60 & 61 & 62 & 63 \\
\hline 48 & 49 & 50 & 51 & 52 & 53 & 54 & 55 \\
\hline 40 & 41 & 42 & 43 & 44 & 45 & 46 & 47 \\
\hline 32 & 33 & 34 & 35 & 36 & 37 & 38 & 39 \\
\hline 24 & 25 & 26 & 27 & 28 & 29 & 30 & 31 \\
\hline 16 & 17 & 18 & 19 & 20 & 21 & 22 & 23 \\
\hline 8 & 9 & 10 & 11 & 12 & 13 & 14 & 15 \\
\hline 0 & 1 & 2 & 3 & 4 & 5 & 6 & 7 \\
\hline
\end{tabular}




\section{Index Spaces}

$\square$ exclusive

$\square$ shared

$\square$ ghost

\begin{tabular}{|c|c|c|c|c|c|c|c|}
\hline 56 & 57 & 58 & 59 & 60 & 61 & 62 & 63 \\
\hline 48 & 49 & 50 & 51 & 52 & 53 & 54 & 55 \\
\hline 40 & 41 & 42 & 43 & 44 & 45 & 46 & 47 \\
\hline 32 & 33 & 34 & 35 & 36 & 37 & 38 & 39 \\
\hline 24 & 25 & 26 & 27 & 28 & 29 & 30 & 31 \\
\hline 16 & 17 & 18 & 19 & 20 & 21 & 22 & 23 \\
\hline 8 & 9 & 10 & 11 & 12 & 13 & 14 & 15 \\
\hline 0 & 1 & 2 & 3 & 4 & 5 & 6 & 7 \\
\hline
\end{tabular}




\section{Cell Partition Information}

exclusive cells

$\begin{array}{lll}0 & 0 & 0\end{array}$ [ ]

101 []

804 []

905 []

1607 []

1708 [ ] shared cells

$\begin{array}{llll}2 & 0 & 2 & \text { [1] }\end{array}$

$\begin{array}{llllll}3 & 0 & 3 & \text { [ } 1]\end{array}$

1006 [ 112 ]

1809 [ 12 ]

24010 [ 3 ]

25011 [ 3 ]

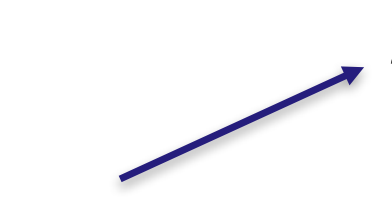

mesh id
26012 [ 23 ]

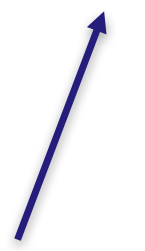

rank local id

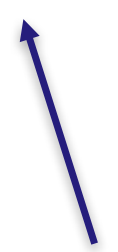

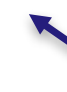

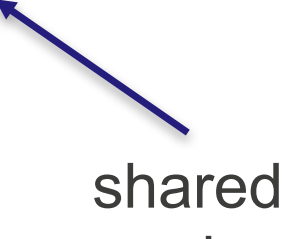
ranks ghost cells

410 [ ]

1114 [ ]

1215 [ ]

1920 [ ]

2721 []

3230 []

3331 [ ]

3432 [ ]

3525 [ ] 


\section{Forming the vertex dependency closure...}

// We can iteratively add halos of nearest neighbors, e.g.,

// here we add the next nearest neighbors. For most mesh types

// we actually need information about the ownership of these indices

// so that we can deterministically assign rank ownership to vertices.

auto nearest_neighbor_closure =

flecsi::topology::entity_closure<2,2,0>(gd, nearest_neighbors);

// The union of the nearest and next-nearest neighbors gives us all

// of the cells that might reference a vertex that we need.

auto all_neighbors = flecsi::utils::set_union(nearest_neighbors, next_nearest_neighbors); 


\section{Rank 0: nearest neighbors + next nearest neighbors}

$\square \quad$ rank 0
$\square \quad$ rank 1
$\square \quad$ rank 2
$\square \quad$ rank 3
$\square \quad$ rank 4

\begin{tabular}{|c|c|c|c|c|c|c|c|}
\hline 56 & 57 & 58 & 59 & 60 & 61 & 62 & 63 \\
\hline 48 & 49 & 50 & 51 & 52 & 53 & 54 & 55 \\
\hline 40 & 41 & 42 & 43 & 44 & 45 & 46 & 47 \\
\hline 32 & 33 & 34 & 35 & 36 & 37 & 38 & 39 \\
\hline 24 & 25 & 26 & 27 & 28 & 29 & 30 & 31 \\
\hline 16 & 17 & 18 & 19 & 20 & 21 & 22 & 23 \\
\hline 8 & 9 & 10 & 11 & 12 & 13 & 14 & 15 \\
\hline 0 & 1 & 2 & 3 & 4 & 5 & 6 & 7 \\
\hline
\end{tabular}




\section{Rank 0: primary + nearest neighbors + next nearest} neighbors

$\square$ rank 0

rank 1

rank 2

rank 3

rank 4

\begin{tabular}{|c|c|c|c|c|c|c|c|}
\hline 56 & 57 & 58 & 59 & 60 & 61 & 62 & 63 \\
\hline 48 & 49 & 50 & 51 & 52 & 53 & 54 & 55 \\
\hline 40 & 41 & 42 & 43 & 44 & 45 & 46 & 47 \\
\hline 32 & 33 & 34 & 35 & 36 & 37 & 38 & 39 \\
\hline 24 & 25 & 26 & 27 & 28 & 29 & 30 & 31 \\
\hline 16 & 17 & 18 & 19 & 20 & 21 & 22 & 23 \\
\hline 8 & 9 & 10 & 11 & 12 & 13 & 14 & 15 \\
\hline 0 & 1 & 2 & 3 & 4 & 5 & 6 & 7 \\
\hline
\end{tabular}




\section{Rank 0 vertex closure}

$\square$ rank 0

rank 1

rank 2

rank 3

rank 4

\begin{tabular}{|c|c|c|c|c|c|c|c|}
\hline 56 & 57 & 58 & 59 & 60 & 61 & 62 & 63 \\
\hline 48 & 49 & 50 & 51 & 52 & 53 & 54 & 55 \\
\hline 40 & 41 & 42 & 43 & 44 & 45 & 46 & 47 \\
\hline $\begin{array}{l}46 \\
32\end{array}$ & $\begin{array}{c}47 \\
33\end{array}$ & $\begin{array}{c}48 \\
34\end{array}$ & $\begin{array}{c}49 \\
35\end{array}$ & 36 & 37 & 38 & 39 \\
\hline $24^{37}$ & $\begin{array}{l}38 \\
25\end{array}$ & $\begin{array}{c}39 \\
26^{39}\end{array}$ & $\begin{array}{r}40 \\
27^{40}\end{array}$ & 28 & 29 & 30 & 31 \\
\hline $\begin{array}{c}28 \\
16\end{array}$ & $17^{\mathbf{2 9}}$ & $18^{30}$ & $\begin{array}{r}31 \\
19\end{array}$ & 20 & 21 & 22 & 23 \\
\hline $8^{19}$ & $9^{20}$ & $10^{\mathbf{2 1}}$ & $\begin{array}{c}\mathbf{2 2} \\
11\end{array}$ & 12 & 13 & 14 & 15 \\
\hline $0^{10}$ & $1^{11}$ & $2^{12}$ & $3^{13}$ & $4^{14}$ & 5 & 6 & 7 \\
\hline
\end{tabular}




\section{Rank 0 vertex closure partitioning}

exclusive

shared

ghost

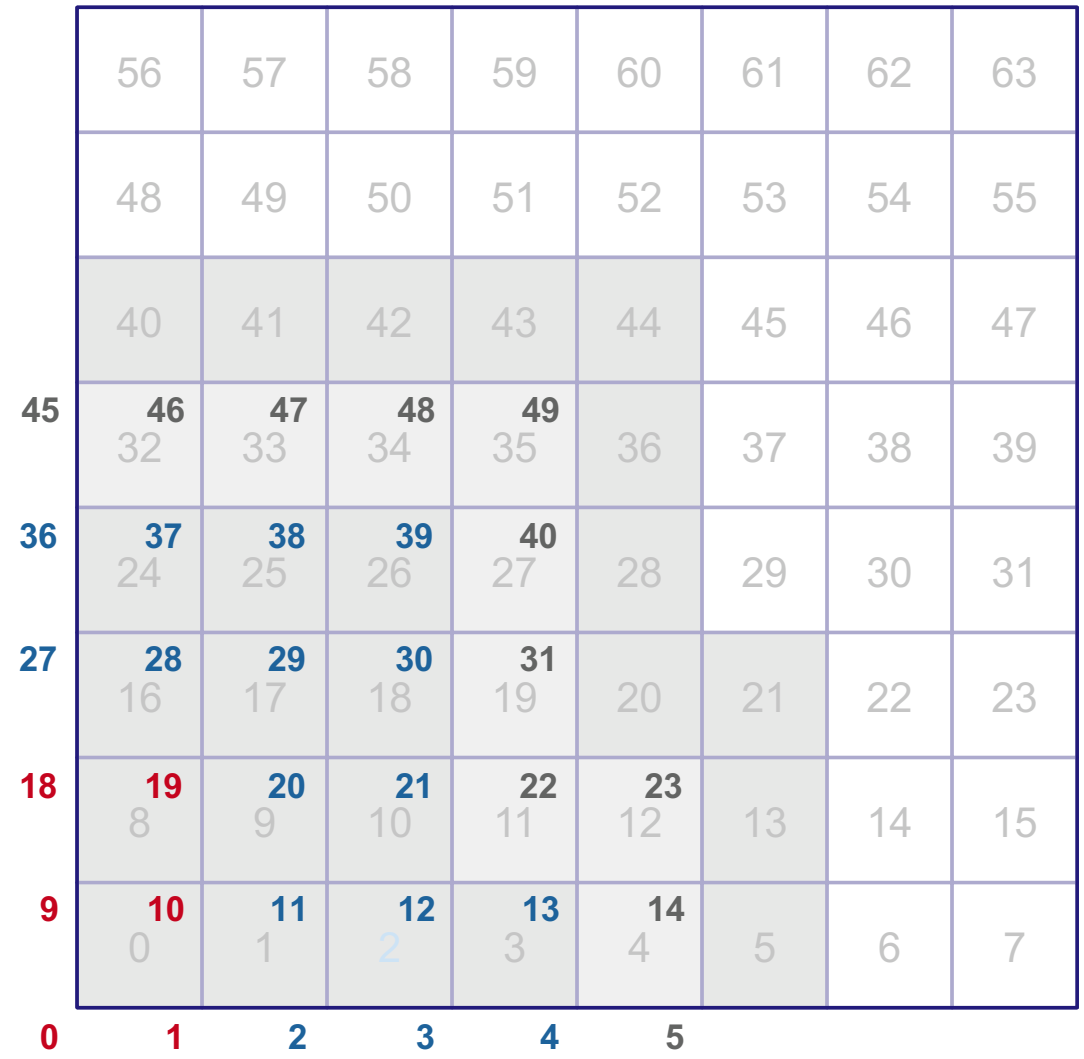




\section{Vertex Partition Information}

exclusive vertices

$\begin{array}{lll}0 & 0 & 0\end{array}$ [ ]

101 [ ]

905 []

1006 []

18010 [ ]

19011 [ ] shared vertices

202 [1]

303 [ 1 ]

404 [1]

1107 [ 12 ]

1208 [ 12 ]

1309 [1]

20012 [ 12 ]

21013 [ 12 ]

27014 [ 3 ]

28015 [ 3 ]

29016 [ [ llll 123 ]

30017 [ [ 1123 ]

36018 [ 3 ]

37019 [ 3 ]

38020 [ 23 ] ]

39021 [ 23 ] ghost vertices

510 []

1414 []

2218 []

2319 [ ]

31113 [ ]

4020 [ ]

4530 []

4631 []

4732 []

4824 []

4925 [ ] 


\section{Simple Partition Examples}



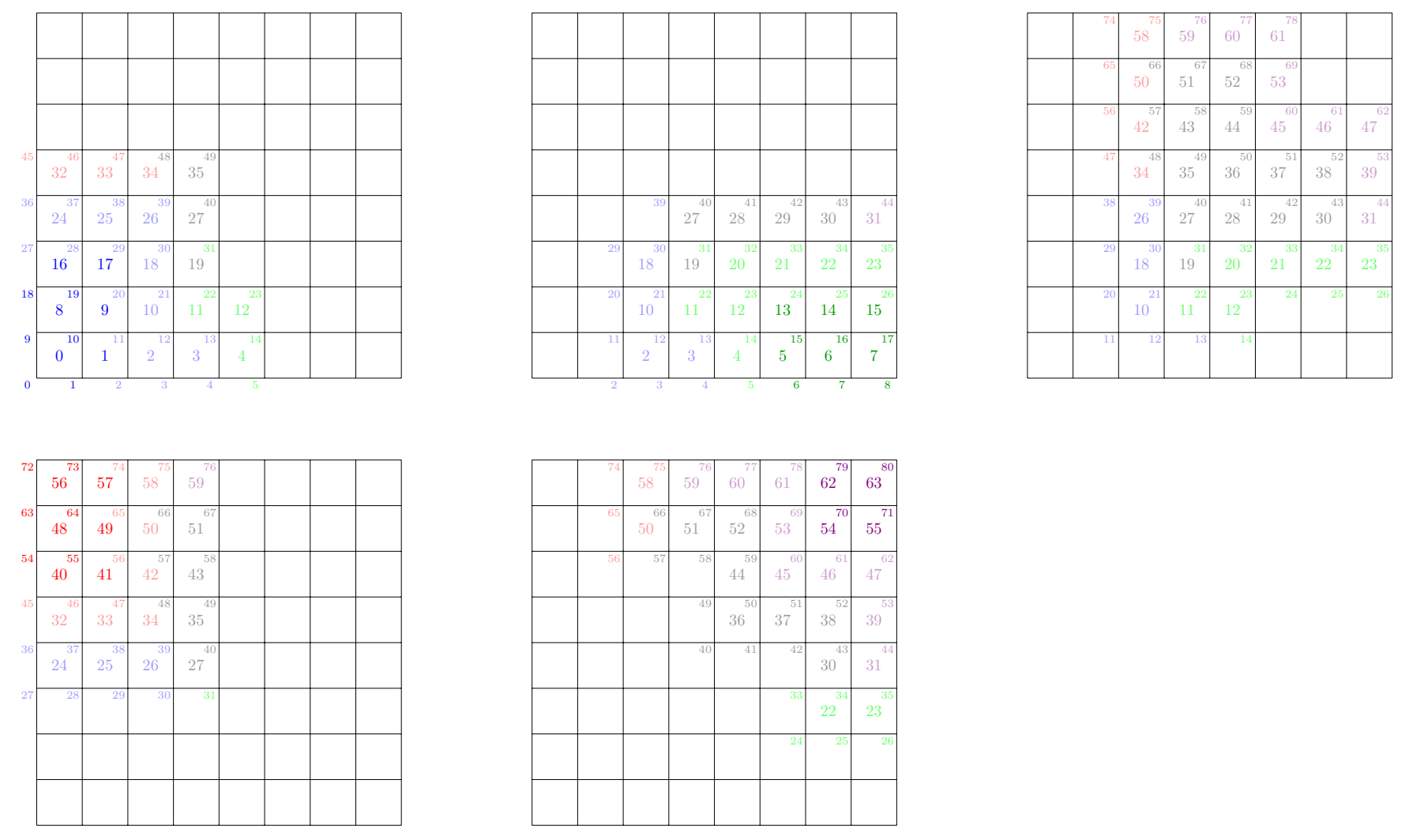

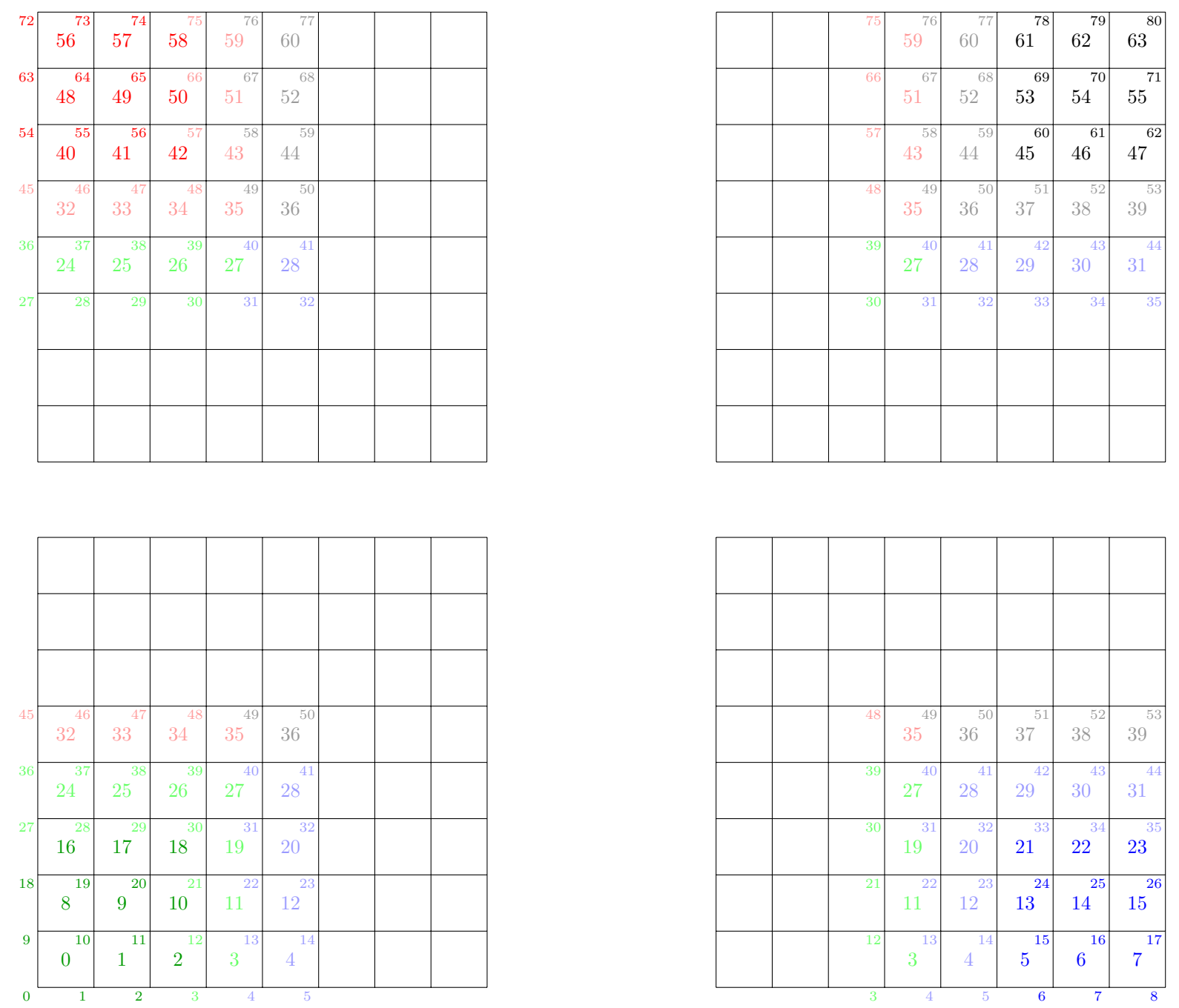